\title{
CYTOSTATIC AND ANTIVIRAL 6-ARYLPURINE RIBONUCLEOSIDES IX.+ SYNTHESIS AND EVALUATION OF 6-SUBSTITUTED 3-DEAZAPURINE RIBONUCLEOSIDES
}

\author{
Petr NAUŠ, Martin KUCHǍ̌ and Michal HOCEK ${ }^{1, *}$ \\ Institute of Organic Chemistry and Biochemistry, Academy of Sciences of the Czech Republic, v.v.i., \\ Gilead Sciences \& IOCB Research Center, Flemingovo nám. 2, 16610 Prague 6, Czech Republic; \\ e-mail: ${ }^{1}$ hocek@uochb.cas.cz
}

Received June 5, 2008

Accepted June 30, 2008

Published online July 25, 2008

Dedicated to the memory of Professor Otto Exner.

A series of 3-deazapurine ribonucleosides $\mathbf{5 a - 5}$ l bearing diverse C-substituents (alkyl, aryl and heteroaryl) in the position 6 were prepared by Pd-catalyzed cross-coupling reactions of either free 6-chloro-3-deazapurine ribonucleoside $\mathbf{4}$ or its acetyl protected congener $\mathbf{3}$ followed by deprotection. An improved synthesis of the starting 4-chloro-1-(2,3,5-tri-0-acetyl$\beta$-D-ribofuranosyl)-1H-imidazo[4,5-c]pyridine (3) was developed by the application of Vorbrüggen glycosylation of silylated nucleobase with 1,2,3,5-tetra-O-acetyl- $\beta$-D-ribofuranose (2). None of compounds 5a-5I showed any considerable cytostatic or antiviral activity.

Keywords: Purines; Imidazo[4,5-c]pyridines; 3-Deazapurines; Nucleosides; Glycosidations; Cross-coupling reactions; Cytostatic activity.

Purine nucleosides bearing aryl or hetaryl substituents in position 6 are cytostatic ${ }^{1}$. Moreover, some 6-hetarylpurine ribonucleosides also exert strong anti-HCV activities ${ }^{2}$. However, the cytotoxic or cytostatic side effect prevents clinical applications as anti-HCV drugs. Therefore, in order to achieve selective inhibition of HCV RNA polymerase, some additional modifications were pursued. From the previous studies on sugar-modified derivatives it is known 2'- and 5'-deoxyribonucleosides ${ }^{3}, 3^{\prime}$-deoxyribonucleosides ${ }^{4}$, as well as 2'-C-methylribonucleosides ${ }^{5}$ of the 6-aryl- or 6-hetarylpurine series are all inactive, while some carbocyclic homonucleosides

+ For Part VIII, see ref. ${ }^{4}$ 
were reported ${ }^{6}$ to still exert some cytostatic effects. Very recently, some L-ribonucleosides were found ${ }^{7}$ to exert weak anti-HCV effect in replicon assay but their triphosphates did not inhibit HCV RNA polymerase. Also modifications of purine ring have been pursued to show that most 2-substituted ${ }^{8}$ and 8 -substituted ${ }^{9}$ 6-aryl purine ribonucleosides were inactive, while some 6-aryl-1-deazapurine nucleosides ${ }^{10}$ still exerted some activities. This shows that the N-1 nitrogen is not crucial for the interaction of these compounds with the target biological system (presumably RNA polymerase and the complementary nucleobase). Therefore the next logical step was to look into the role of $\mathrm{N}-3$ nitrogen which does not make $\mathrm{H}$-bonds with the complementary pyrimidine nucleobase during biosynthesis of RNA but is responsible for crucial minor groove interactions in the active site of the polymerase. In this paper we report on the synthesis and evaluation of cytostatic and anti-HCV activity of novel 6-aryl-3-deazapurine ribonucleosides. Taking into account also known cytostatic activities of 6-methylpurine ${ }^{11}$ and recently reported 6-cyclopropylpurine ribonucleosides $^{12}$, the series of 6-aryl and hetaryl derivatives was also complemented by examples of 6-alkyl-3-deazapurine nucleosides.
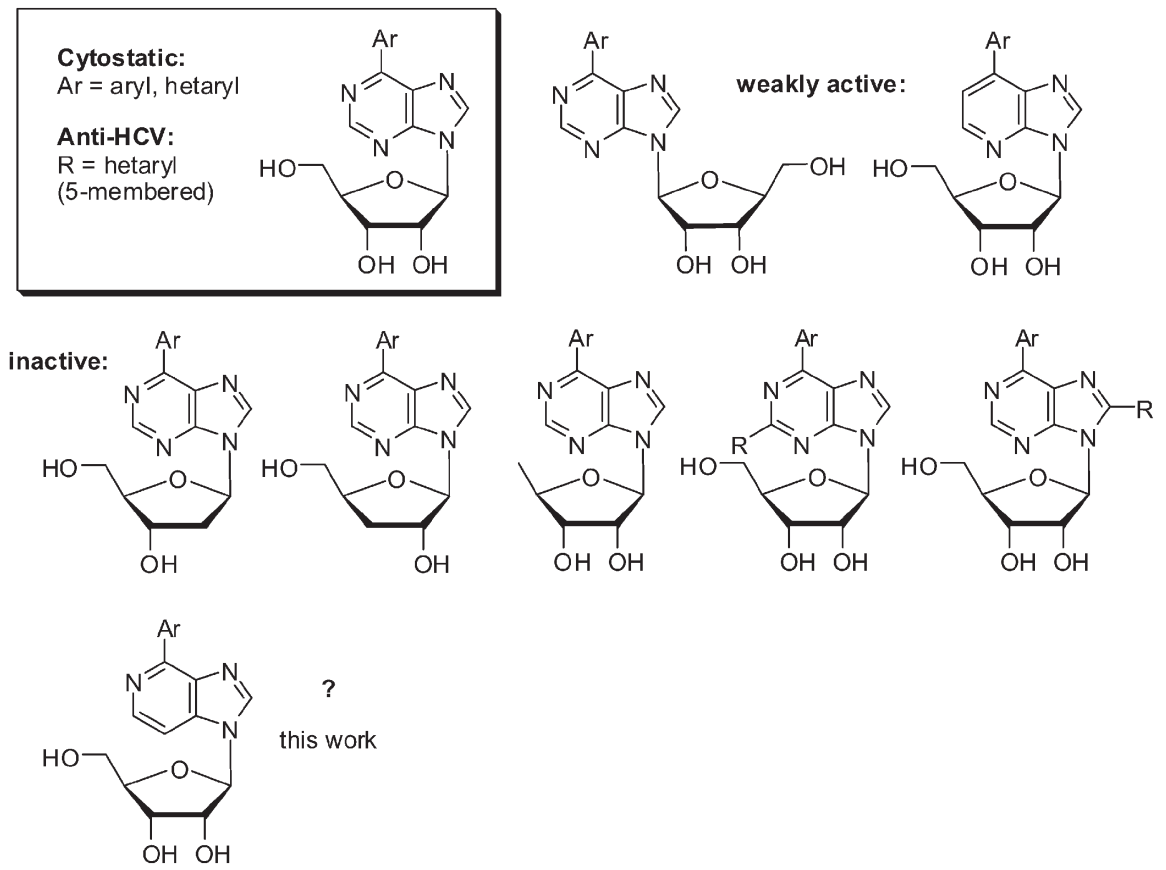
3-Deazaadenosine ( $c^{3} \mathrm{~A}$, 4-aminoimidazo[4,5-c]pyridine) and its analogues are substrates and potent inhibitors of S-adenosyl-L-homocysteine hydrolase $^{13}$ and subsequent perturbation of transmethylation reactions is at least partly responsible for their diverse biological effects. These compounds exert antiviral ${ }^{14}$, cytotoxic $^{15}$, tuberculostatic ${ }^{16}$ immunosuppressive and antiinflammatory properties ${ }^{17}$. No 3-deazapurine bearing a $\mathrm{C}$-substituent in position 6 was reported to the best of our knowledge.

\section{RESULTS AND DISCUSSION}

At first we had to prepare either protected or free 6-chloro-3-deazapurine riboside intermediates as starting compounds for Pd-catalyzed crosscoupling reactions with aryl(hetaryl)organometallics and boronic acids. To our surprise all the known syntheses of these nucleosides based on glycosylation of 6-chloro-3-deazapurine $\mathbf{1}$ rely either on mercury salt method $^{18}$ or fusion method ${ }^{19}$ both suffering from low overall yield, need for excess of glycosyl component, complicated separation from by-products and possible contamination by mercury salts. Therefore we were attracted by the application of the Hilbert-Johnson reaction performed under Vorbrüggen conditions ${ }^{20}$ as this approach was recently successfully employed for 3,6-difluoro-3-deazapurine ${ }^{21}$. Thus 4-chloroimidazo[4,5-c]pyridine $^{22}$ (1) was silylated by treatment with BSA in acetonitrile and then reacted with 1,2,3,5-tetra-O-acetyl- $\beta$-D-ribofuranose (2) in presence of TMSOTf at $80{ }^{\circ} \mathrm{C}$ for $1 \mathrm{~h}$ to afford the desired crystalline acetylated nucleoside 3 in $89 \%$ yield (Scheme 1). Deprotection of $\mathbf{3}$ by treatment with methanolic ammonia at room temperature for $24 \mathrm{~h}$ afforded desired free nucleoside 4 in $86 \%$ yield after crystallization.

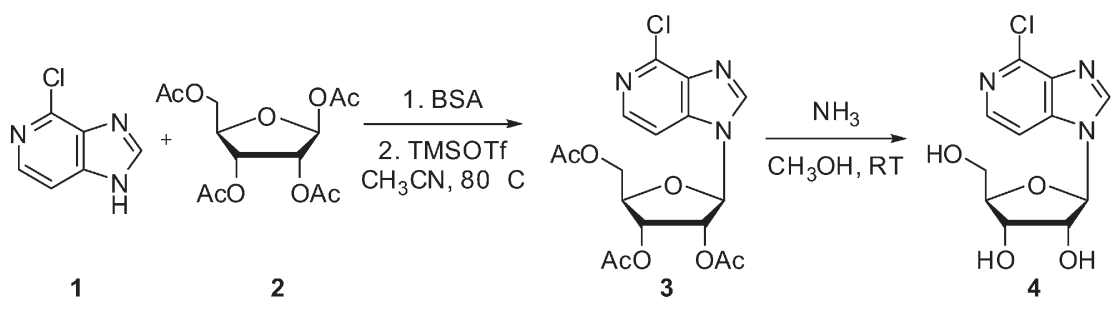

SCHEME 1 
TABLE I

Suzuki-Miyaura reaction of chloride $\mathbf{4}$ with boronic acids under Shaughnessy conditions
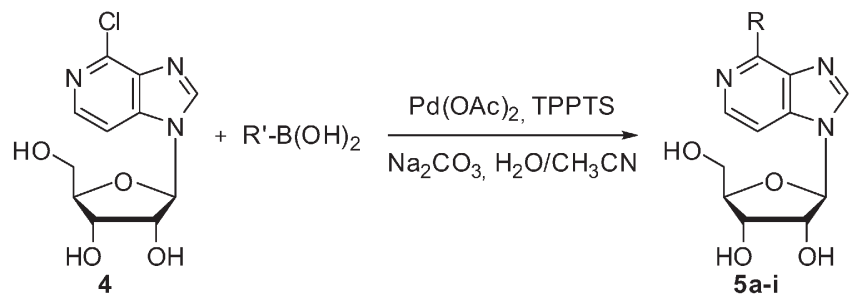

Entry

$\mathrm{R}^{\prime}-\mathrm{B}(\mathrm{OH})_{2}$

$\mathrm{R}$

Reaction time, $\mathrm{h}$
Cross-Coupling Product (yield)
1<smiles>Oc1ccccc1</smiles>

2

3

4

5

6<smiles>Oc1ccco1</smiles><smiles>Oc1cccs1</smiles><smiles>O[Se]c1ccoc1</smiles><smiles>Oc1ccsc1</smiles><smiles></smiles>

7<smiles>C[SiH2][SiH2]n1ccc([Hg]O)c1</smiles>

$8^{\mathrm{a}}$<smiles>O[Ga]c1ccn[nH]1</smiles>

9<smiles>Oc1cccnc1</smiles>

3

5a (92\%)

5b (88\%)

3

5c $(90 \%)$

2

5d (72\%)

$5 e(78 \%)$

24

$5 f(50 \%)$<smiles>Cc1ccc[nH]1</smiles><smiles>Cc1cc[nH]c1</smiles>

5g (61\%)

24

5h $(10 \%)^{\mathrm{a}}$

20

a $82 \%$ of starting material 4 recovered. 
With starting compounds in hands we have performed cross-coupling reactions leading to our desired 6-substituted derivatives. At first, we attempted aqueous Suzuki-Miyaura reactions of free ribonucleoside $\mathbf{4}$ under the Shaughnessy conditions ${ }^{23,24}$. The treatment of 6-chloro-3-deazapurine riboside 4 with diverse aryl- or hetarylboronic acids in the presence of $\mathrm{Pd}(\mathrm{OAC})_{2}$, TPPTS, and $\mathrm{Na}_{2} \mathrm{CO}_{3}$ in $\mathrm{H}_{2} \mathrm{O}-\mathrm{CH}_{3} \mathrm{CN}(2: 1)$ at $100{ }^{\circ} \mathrm{C}$ provided desired 6-aryl(hetaryl)-3-deazapurine ribosides 5a-5i (Table I). The reactions proceeded smoothly and cleanly for phenyl-, 3-pyridyl- and for 2- and 3furyl- and thienylboronic acids, providing the desired products in high yields (entries 1-5, 9). For 2-pyrrolyl and 3-pyrrolyl the isolated yields of products $\mathbf{5} \mathbf{f}$ and $\mathbf{5 g}$ were only moderate (entries 6,7 ), but all starting chloride 4 was al ways fully consumed indicating possible partial decomposition of products $\mathbf{5} \mathbf{f}$ and $\mathbf{5 g}$ under reaction conditions. It should be also noted, that $\mathrm{N}$-protecting groups in both starting pyrrolylboronic acids were simultaneously removed under the conditions of coupling (BOC for 2-pyrrolyl and triisopropylsilyl for 3-pyrrolyl). The reaction of $\mathbf{4}$ with $1 \mathrm{H}$-pyrazole5-boronic acid was very sluggish giving product $\mathbf{5 h}$ (entry 8 ) in only $10 \%$ yield after crystallization and $82 \%$ of starting chloride 4 was recovered.

While the attempts to introduce similarly methyl and ethyl substituents by Suzuki reaction of $\mathbf{4}$ with corresponding alkylboronic acids under above mentioned conditions failed (in the case of methyl only trace of the product was obtained and for ethyl no reaction was observed) we turned to reactions with trialkylaluminums known ${ }^{25}$ to be suitable for introduction of alkyl substituetnts to purines. Thus acetylated 6-chloro-3-deazapurine riboside $\mathbf{3}$ was reacted with trimethyl- and triethylaluminums in the presence of $\mathrm{Pd}\left(\mathrm{PPh}_{3}\right)_{4}$ in refluxing THF affording 6-alkylpurine nucleosides $\mathbf{6 a}$ and $\mathbf{6 b}$ in 53 and $60 \%$ yields, respectively (Scheme 2). These acetylated products $\mathbf{6 a}$ and $\mathbf{6 b}$ were deprotected with catalytic sodium methoxide in methanol giving the desired nucleosides $\mathbf{5} \mathbf{j}$ and $\mathbf{5 k}$ in 71 and $70 \%$ yields, re-

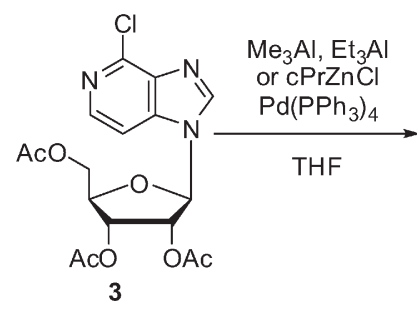

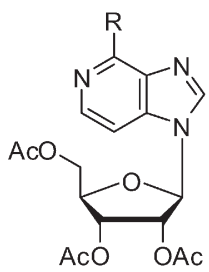

$6 a(R=M e, 53 \%)$

6b $(\mathrm{R}=\mathrm{Et}, 60 \%)$

$6 \mathbf{c}(\mathrm{R}=\mathrm{cPr}, 38 \%)$

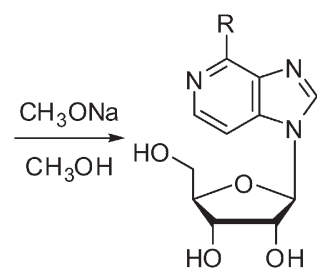

$5 \mathrm{j}(\mathrm{R}=\mathrm{Me}, 71 \%)$

$5 \mathbf{k}(\mathrm{R}=\mathrm{Et}, 70 \%)$

5I $(\mathrm{R}=\mathrm{cPr}, 70 \%)$

SCHEME 2 
spectively, after recrystallization. For the preparation cyclopropyl derivative Negishi reaction between chloride 3 and cyclopropylzinc chloride in the presence of $\mathrm{Pd}\left(\mathrm{PPh}_{3}\right)_{4}$ was conducted to furnish protected cyclopropyl derivative $\mathbf{6 c}$ in moderate $38 \%$ yield and subsequent deprotection gave free nucleoside 5I (70\%).

It should be noted, that some of the attempted cross-couplings (successful in 6-chloropurines) failed here with 6-chloro-3-deazapurines $\mathbf{3}$ or $\mathbf{4}$. The Stille reaction of acetylated nucleoside $\mathbf{3}$ with 2-(tributylstannyl)thiazole or 2-(tributylstannyl)pyridine and the Negishi reaction with benzoyloxymethylzinc iodide ${ }^{26}$ can serve as example. Markedly decreased reactivity of 3-deazapurine derivatives compared to corresponding purines in nucleophilic substitutions of 6-chloro group is known ${ }^{19}$.

All the title 6-substituted 3-deazapurine ribonucleosides 5a-5I were subjected to biological activity screening. The cytostatic activity in vitro (inhibition of cell growth) was studied on the following cell cultures: mouse leukemia L1210 cells (ATCC CCL 219), human promyelocytic leukemia HL60 cells (ATCC CCL 240), human cervix carcinoma HeLaS3 cells (ATCC CCL 2.2) and human T lymphoblastoid CCRF-CEM cell line (ATCC CCL 119). Antiviral activity of nucleosides 5a-5l was evaluated in a HCV subgenomic replicon assay ${ }^{27}$. None of the compounds showed any considerable cytostatic or antiviral activity in these assays up to $10 \mu \mathrm{M}$ concentration. Apparently, removal of the N-3 nitrogen leads to inactive compounds indicating that some specific interactions of this nitrogen with the target biological system (most probably minor groove $\mathrm{H}$-bond interaction in the active site of the RNA polymerase) are crucial for the biological activity of this class of compounds.

\section{EXPERIMENTAL}

NMR spectra were recorded on Bruker Avance $400 \mathrm{MHz}\left({ }^{1} \mathrm{H}\right.$ at $400 \mathrm{M} \mathrm{Hz},{ }^{13} \mathrm{C}$ at $\left.100.6 \mathrm{M} \mathrm{Hz}\right)$ and Bruker Avance $500 \mathrm{M} \mathrm{Hz}\left(500 \mathrm{M} \mathrm{Hz}\right.$ for ${ }^{1} \mathrm{H}$ and $125.7 \mathrm{M} \mathrm{Hz}$ for ${ }^{13} \mathrm{C}$ ) spectrometers. Chemical shifts (in ppm, $\delta$-scale) were referenced to TMS as internal standard. Coupling constants (J) are given in $\mathrm{Hz}$. The assignment of carbons was based on $\mathrm{C}, \mathrm{H}-\mathrm{HSQC}$ and $\mathrm{C}, \mathrm{H}-\mathrm{HMBC}$ experiments. IR spectra (wavenumbers in $\mathrm{cm}^{-1}$ ) were recorded on a Brucker IFS 88 spectrometer. Melting points were determined on a Kofler block and are uncorrected. Optical rotations were measured at $25{ }^{\circ} \mathrm{C}$ on an Autopol IV (Rudolph Research Analytical) polarimeter, $[\alpha]_{D}$ values are given in $10^{-1} \mathrm{deg} \mathrm{cm}^{2} \mathrm{~g}^{-1}$. High resolution mass spectra (HR MS) were measured on a LTQ Orbitrap XL (Thermo Fisher Scientific) spectrometer using electrospray ionization. High performance flash chromatography (HPFC) purifications were performed using SP1 ${ }^{\text {TM }}$ Flash Purification System (Biotage) on C-18 columns using water-methanol gradient. THF was dried and freshly distilled from sodium/benzophenone. 
4-Chloro-1-(2,3,5-tri-O-acetyl- $\beta$-D-ribofuranosyl)-1H-imidazo[4,5-c]pyridine (3)

To a slurry of 4-chloro-1H-imidazo[4,5-c]pyridine ${ }^{22}(\mathbf{1} ; 1.69 \mathrm{~g}, 11 \mathrm{mmol})$ in dry $\mathrm{CH}_{3} \mathrm{CN}(50 \mathrm{ml})$, BSA $(2.72 \mathrm{ml}, 11 \mathrm{mmol}$ ) was added and the mixture was stirred at RT for $20 \mathrm{~min}$ (clear solution), followed by addition of tetracetyl ribose 2 (3.85 g, $12.1 \mathrm{mmol})$. TMSOTf (1.99 ml, $11 \mathrm{mmol}$ ) was then added dropwise at $0{ }^{\circ} \mathrm{C}$ and the mixture was heated at $80{ }^{\circ} \mathrm{C}$ for $3 \mathrm{~h}$. After cooling and dilution with $\mathrm{CHCl}_{3}(100 \mathrm{ml})$ the mixture was washed with saturated aqueous $\mathrm{NaHCO}_{3}(50 \mathrm{ml})$. Aqueous phase was reextracted with $\mathrm{CHCl}_{3}(2 \times 10 \mathrm{ml})$, collected organics were dried over $\mathrm{MgSO}_{4}$ and final column chromatography on silica (hexanes-AcOEt, 1:1) provided nucleoside 3 (4.05 g, 89\%) as a colorless crystalline solid, m.p. 160-161 ${ }^{\circ} \mathrm{C}$ (hexane-AcOEt); ref. ${ }^{19} 158.5-159{ }^{\circ} \mathrm{C} .{ }^{1} \mathrm{H} \mathrm{NMR}\left(400 \mathrm{MHz} \mathrm{CDCl}_{3}\right): 2.10,2.15$, $2.16\left(3 \times \mathrm{s}, 3 \times 3 \mathrm{H}, \mathrm{CH}_{3} \mathrm{CO}\right) ; 4.39\left(\mathrm{dd}, 1 \mathrm{H}, \mathrm{J}_{\mathrm{gem}}=12.6, \mathrm{~J}_{5^{\prime} \mathrm{b}, 4^{\prime}}=2.7, \mathrm{H}-5^{\prime} \mathrm{b}\right) ; 4.46(\mathrm{dd}, 1 \mathrm{H}$, $\left.\mathrm{J}_{\text {gem }}=12.6, \mathrm{~J}_{5^{\prime} \mathrm{a}, 4^{\prime}}=2.9, \mathrm{H}-5^{\prime} \mathrm{a}\right) ; 4.51\left(\mathrm{ddd}, 1 \mathrm{H}, \mathrm{J}_{4^{\prime}, 3^{\prime}}=5.1, \mathrm{~J}_{4^{\prime}, 5^{\prime}}=2.9,2.7, \mathrm{H}-4^{\prime}\right) ; 5.38(\mathrm{dd}, 1 \mathrm{H}$, $\left.\mathrm{J}_{3^{\prime}, 2^{\prime}}=5.3, \mathrm{~J}_{3^{\prime}, 4^{\prime}}=5.1, \mathrm{H}-3^{\prime}\right) ; 5.51\left(\mathrm{t}, 1 \mathrm{H}, \mathrm{J}_{2^{\prime}, 1^{\prime}}=5.3, \mathrm{~J}_{2^{\prime}, 3^{\prime}}=5.3, \mathrm{H}-2^{\prime}\right) ; 6.07\left(\mathrm{~d}, 1 \mathrm{H}, \mathrm{J}_{1^{\prime}, 2^{\prime}}=5.3\right.$, $\left.\mathrm{H}-1^{\prime}\right) ; 7.53\left(\mathrm{~d}, 1 \mathrm{H}, \mathrm{J}_{7,6}=5.6, \mathrm{H}-7\right) ; 8.23\left(\mathrm{~d}, 1 \mathrm{H}, \mathrm{J}_{6,7}=5.6, \mathrm{H}-6\right) ; 8.27(\mathrm{~s}, 1 \mathrm{H}, \mathrm{H}-2)$. ${ }^{13} \mathrm{C} \mathrm{NMR}$ (100.6 $\left.\mathrm{MHz} \mathrm{CDCl}_{3}\right)$ : 20.32, 20.45 and $20.73\left(3 \times \mathrm{CH}_{3} \mathrm{CO}\right) ; 62.46\left(\mathrm{CH}_{2}-5^{\prime}\right) ; 69.81\left(\mathrm{CH}-3^{\prime}\right)$; $73.53\left(\mathrm{CH}-2^{\prime}\right) ; 80.54\left(\mathrm{CH}-4^{\prime}\right) ; 87.44\left(\mathrm{CH}^{\prime} \mathbf{1}^{\prime}\right) ; 105.94(\mathrm{CH}-7) ; 138.25$ (C-7a); 138.63 (C-3a); $141.78(\mathrm{CH}-2) ; 142.08(\mathrm{CH}-6) ; 143.34(\mathrm{C}-4) ; 169.27,169.46$ and $170.00(3 \times \mathrm{CO}) . \mathrm{IR}\left(\mathrm{CCl}_{4}\right)$ : 2959, 2927, 2855, 1759, 1234, 1219, 1211, 967. HR MS (ESI): calculated for $\mathrm{C}_{17} \mathrm{H}_{18} \mathrm{CIN}_{3} \mathrm{NaO}_{7}$ [M + Na] 434.0731, found 434.0727. For $\mathrm{C}_{17} \mathrm{H}_{18} \mathrm{CIN}_{3} \mathrm{O}_{7}$ calculated: $49.58 \% \mathrm{C}, 4.41 \% \mathrm{H}$, $10.20 \% \mathrm{~N}$; found: $49.41 \% \mathrm{C}, 4.38 \% \mathrm{H}, 10.04 \% \mathrm{~N}$.

\section{4-Chloro-1-( $\beta$-D-ribofuranosyl)-1H-imidazo[4,5-c]pyridine (4)}

Acetylated nucleoside 3 (3.29 g, $7.99 \mathrm{mmol})$ was treated with methanolic ammonia (24\%, $70 \mathrm{ml}$ ) at RT for $24 \mathrm{~h}$. After removal of volatiles under reduced pressure, the product was crystallized from $96 \%$ ethanol affording chloro riboside $4(1.96 \mathrm{~g}, 86 \%)$ as colorless prisms, m.p. $192-193{ }^{\circ} \mathrm{C}$; ref. ${ }^{19} 192.5-193.5{ }^{\circ} \mathrm{C}$. $[\alpha]_{D}-39.1$ (c 0.13 , DMSO); ref. ${ }^{18}[\alpha]_{D}-39.1$ (c 1.02, $\mathrm{MeOH})$; ref. ${ }^{19}[\alpha]_{D}-41.6(\mathrm{c} 1.25, \mathrm{MeOH}) .{ }^{1} \mathrm{H} \mathrm{NMR}\left(400 \mathrm{MHz}, \mathrm{DMSO}-\mathrm{d}_{6}\right): 3.64$ (ddd, $1 \mathrm{H}$, $\left.\mathrm{J}_{\mathrm{gem}}=12.0, \mathrm{~J}_{5^{\prime} \mathrm{b}, \mathrm{OH}}=5.1, \mathrm{~J}_{5^{\prime} \mathrm{b}, 4^{\prime}}=3.5, \mathrm{H}-5^{\prime} \mathrm{b}\right) ; 3.69\left(\mathrm{ddd}, 1 \mathrm{H}, \mathrm{J}_{\mathrm{gem}}=12.0, \mathrm{~J}_{5^{\prime} \mathrm{a}, \mathrm{OH}}=5.3, \mathrm{~J}_{5^{\prime} \mathrm{a}, 4^{\prime}}=\right.$ 3.5, H-5'a); 4.01 (td, $\left.1 \mathrm{H}, \mathrm{J}_{4^{\prime}, 5^{\prime}}=3.5, \mathrm{~J}_{4^{\prime}, 3^{\prime}}=3.0, \mathrm{H}-4^{\prime}\right) ; 4.12\left(\mathrm{ddd}, 1 \mathrm{H}, \mathrm{J}_{3^{\prime}, 2^{\prime}}=5.1, \mathrm{~J}_{3^{\prime}, \mathrm{OH}}=4.6\right.$, $\left.\mathrm{J}_{3^{\prime}, 4^{\prime}}=3.0, \mathrm{H}-3^{\prime}\right) ; 4.34\left(\mathrm{ddd}, 1 \mathrm{H}, \mathrm{J}_{2^{\prime}, \mathrm{OH}}=6.3, \mathrm{~J}_{2^{\prime}, 1^{\prime}}=6.3, \mathrm{~J}_{2^{\prime}, 3^{\prime}}=5.1, \mathrm{H}-2^{\prime}\right) ; 5.20(\mathrm{dd}, 1 \mathrm{H}$, $\left.\mathrm{J}_{\mathrm{OH}, 5^{\prime} \mathrm{a}}=5.3, \mathrm{~J}_{\mathrm{OH}, 5^{\prime} \mathrm{b}}=5.1, \mathrm{OH}-5^{\prime}\right) ; 5.27\left(\mathrm{~d}, 1 \mathrm{H}, \mathrm{J}_{\mathrm{OH}, 3^{\prime}}=4.6, \mathrm{OH}-3^{\prime}\right) ; 5.54\left(\mathrm{~d}, 1 \mathrm{H}, \mathrm{J}_{\mathrm{OH}, 2^{\prime}}=6.3\right.$, $\left.\mathrm{OH}-2^{\prime}\right) ; 5.93\left(\mathrm{~d}, 1 \mathrm{H}, \mathrm{J}_{1^{\prime}, 2^{\prime}}=6.3, \mathrm{H}-\mathrm{I}^{\prime}\right) ; 7.91\left(\mathrm{~d}, 1 \mathrm{H}, \mathrm{J}_{7,6}=5.5, \mathrm{H}-7\right) ; 8.17\left(\mathrm{~d}, 1 \mathrm{H}, \mathrm{J}_{6,7}=5.6\right.$, $\mathrm{H}-6) ; 8.70(\mathrm{~s}, 1 \mathrm{H}, \mathrm{H}-2) .{ }^{13} \mathrm{C}$ NMR (100.6 M Hz, DMSO-d $): 61.59\left(\mathrm{CH}_{2}-5^{\prime}\right) ; 70.63\left(\mathrm{CH}-3^{\prime}\right)$; 74.69 (CH-2'); $86.62\left(\mathrm{CH}-4^{\prime}\right) ; 89.73\left(\mathrm{CH}^{\prime} \mathbf{1}^{\prime}\right) ; 108.25(\mathrm{CH}-7) ; 138.00$ (C-3a); 139.71 (C-7a); 141.55 (C-4); 141.59 (CH-6); 145.07 (CH-2). IR (KBr): 1604, 1570, 1489, 1445, 1309, 1216, 1202, 1114, 1082, 1043. HR MS (ESI): calculated for $\mathrm{C}_{11} \mathrm{H}_{12} \mathrm{CIN}_{3} \mathrm{NaO}_{4}[\mathrm{M}+\mathrm{Na}] 308.0414$, found 308.0408. For $\mathrm{C}_{11} \mathrm{H}_{12} \mathrm{ClN}_{3} \mathrm{O}_{4}$ calculated: $46.25 \% \mathrm{C}, 4.23 \% \mathrm{H}, 14.71 \% \mathrm{~N}$; found: $46.11 \% \mathrm{C}, 4.26 \% \mathrm{H}, 14.31 \% \mathrm{~N}$.

\section{Preparation of 6-Aryl(hetaryl)-3-deazapurine Ribosides 5a-5i. General Procedure}

To an argon purged flask containing 6-chloro-3-deazapurine riboside 4 ( $214 \mathrm{mg}, 0.75 \mathrm{mmol}$ ), boronic acid $(0.94 \mathrm{mmol})$ and $\mathrm{Na}_{2} \mathrm{CO}_{3}(236 \mathrm{mg}, 2.25 \mathrm{mmol})$, a pre-prepared solution of $\mathrm{Pd}(\mathrm{OAC})_{2}(8 \mathrm{mg}, 0.037 \mathrm{mmol})$ and TPPTS $(53 \mathrm{mg}, 0.093 \mathrm{mmol})$ in water- $\mathrm{CH}_{3} \mathrm{CN}(2: 1,4 \mathrm{ml})$ was added. The reaction mixture was stirred at $100{ }^{\circ} \mathrm{C}$ for $2-24 \mathrm{~h}$. After cooling the mixture 
was neutralized by the addition of aqueous $\mathrm{HCl}$ ( $3 \mathrm{~m}$ solution) and after concentration in vacuo final purification by reverse phase chromatography afforded products $\mathbf{5 a - 5 i}$.

4-Phenyl-1-( $\beta$-D-ribofuranosyl)-1H-imidazo[4,5-c]pyridine (5a). Yield 92\%. Reaction time $3 \mathrm{~h}$. White solid after lyophilization, m.p. $99-104^{\circ} \mathrm{C}$. $[\alpha]_{\mathrm{D}}-59.5$ (c 1.6, DMSO). ${ }^{1} \mathrm{H}$ NMR (400 MHz, DMSO $\left.-d_{6}\right): 3.66\left(d d d, 1 \mathrm{H}, \mathrm{J}_{\text {gem }}=12.0, \mathrm{~J}_{5^{\prime} \mathrm{b}, \mathrm{OH}}=5.1, \mathrm{~J}_{5^{\prime} \mathrm{b}, 4^{\prime}}=3.5, \mathrm{H}-5^{\prime} \mathrm{b}\right) ; 3.71$ (ddd, $1 \mathrm{H}$, $\left.\mathrm{J}_{\text {gem }}=12.0 \mathrm{~J}_{5^{\prime} \mathrm{a}, \mathrm{OH}}=5.3, \mathrm{~J}_{5^{\prime} \mathrm{a}, 4^{\prime}}=3.5, \mathrm{H}-5^{\prime} \mathrm{a}\right) ; 4.03\left(\mathrm{td}, 1 \mathrm{H}, \mathrm{J}_{4^{\prime}, 5^{\prime}}=3.5, \mathrm{~J}_{4^{\prime}, 3^{\prime}}=3.1, \mathrm{H}-4^{\prime}\right) ; 4.16$ $\left(\mathrm{ddd}, 1 \mathrm{H}, \mathrm{J}_{3^{\prime}, 2^{\prime}}=5.1, \mathrm{~J}_{3^{\prime}, \mathrm{OH}}=4.7, \mathrm{~J}_{3^{\prime}, 4^{\prime}}=3.1, \mathrm{H}-3^{\prime}\right) ; 4.42\left(\mathrm{ddd}, 1 \mathrm{H}, \mathrm{J}_{2^{\prime}, \mathrm{OH}}=6.4, \mathrm{~J}_{2^{\prime}, 1^{\prime}}=6.3\right.$, $\left.\mathrm{J}_{2^{\prime}, 3^{\prime}}=5.1, \mathrm{H}-2^{\prime}\right) ; 5.21\left(\mathrm{dd}, 1 \mathrm{H}, \mathrm{J}_{\mathrm{OH}, 5^{\prime} \mathrm{a}}=5.3, \mathrm{~J}_{\mathrm{OH}, 5^{\prime} \mathrm{b}}=5.1, \mathrm{OH}-5^{\prime}\right) ; 5.30\left(\mathrm{~d}, 1 \mathrm{H}, \mathrm{J}_{\mathrm{OH}, 3^{\prime}}=4.7\right.$, $\left.\mathrm{OH}-3^{\prime}\right) ; 5.57\left(\mathrm{~d}, 1 \mathrm{H}, \mathrm{J}_{\mathrm{OH}, 2^{\prime}}=6.4, \mathrm{OH}-2^{\prime}\right) ; 5.97\left(\mathrm{~d}, 1 \mathrm{H}, \mathrm{J}_{1^{\prime}, 2^{\prime}}=6.3, \mathrm{H}-1^{\prime}\right) ; 7.42-7.56(\mathrm{~m}, 3 \mathrm{H}$, $\mathrm{H}-\mathrm{m}, \mathrm{p}-\mathrm{Ph}$ ); $7.82\left(\mathrm{~d}, 1 \mathrm{H}, \mathrm{J}_{7,6}=5.5, \mathrm{H}-7\right) ; 8.46\left(\mathrm{~d}, 1 \mathrm{H}, \mathrm{J}_{6,7}=5.5, \mathrm{H}-6\right) ; 8.68-8.72(\mathrm{~m}, 3 \mathrm{H}, \mathrm{H}-2$ and $\mathrm{H}-0-\mathrm{Ph}) .{ }^{13} \mathrm{C}$ NMR (100.6 $\left.\mathrm{MHz}^{-} \mathrm{DMSO}-\mathrm{d}_{6}\right): 61.17\left(\mathrm{CH}_{2}-5^{\prime}\right) ; 70.15\left(\mathrm{CH}-3^{\prime}\right) ; 73.97\left(\mathrm{CH}-2^{\prime}\right)$; 85.89 (CH-4'); $\left.88.87\left(\mathrm{CH}^{\prime}\right)^{\prime}\right) ; 106.50$ (CH-7); 128.19 (CH-m-Ph); 128.91 (CH-o-Ph); 128.97 (CH-p-Ph); 137.62 (C-i-Ph); 138.26 (C-3a); 138.95 (C-7a); 141.43 (CH-6); 143.64 (CH-2); 147.72 (C-4). IR (KBr): 1602, 1585, 1574, 1301, 1221, 1100, 1060. HR MS (ESI): calculated for $\mathrm{C}_{17} \mathrm{H}_{18} \mathrm{~N}_{3} \mathrm{O}_{4}[\mathrm{M}+\mathrm{H}]$ 328.1297, found 328.1295. For $\mathrm{C}_{17} \mathrm{H}_{17} \mathrm{~N}_{3} \mathrm{O}_{4} \cdot 0.7 \mathrm{H}_{2} \mathrm{O}$ calculated: $60.06 \% \mathrm{C}, 5.46 \% \mathrm{H}, 12.36 \% \mathrm{~N}$; found: $60.38 \% \mathrm{C}, 5.23 \% \mathrm{H}, 12.05 \% \mathrm{~N}$.

4-(Furan-2-yl)-1-( $\beta$-D-ribofuranosyl)-1H-imidazo[4,5-c]pyridine (5b). Yield 88\%. Reaction time 3 h. Colorless crystals from water, m.p. $119-122{ }^{\circ} \mathrm{C}$. $[\alpha]_{D}-57.2$ (c 3.5, DMSO). ${ }^{1} \mathrm{H}$ NMR (400 MHz, DMSO $-d_{6}$ ): $3.65\left(d d d, 1 \mathrm{H}, \mathrm{J}_{\text {gem }}=12.0, \mathrm{~J}_{5^{\prime} \mathrm{b}, \mathrm{OH}}=5.1, \mathrm{~J}_{5^{\prime} \mathrm{b}, 4^{\prime}}=3.5, \mathrm{H}-5^{\prime} \mathrm{b}\right) ; 3.71(\mathrm{ddd}, 1 \mathrm{H}$, $\left.\mathrm{J}_{\text {gem }}=12.0, \mathrm{~J}_{5^{\prime} \mathrm{a}, \mathrm{OH}}=5.3, \mathrm{~J}_{5^{\prime} \mathrm{a}, 4^{\prime}}=3.5, \mathrm{H}-5^{\prime} \mathrm{a}\right) ; 4.02\left(\mathrm{td}, 1 \mathrm{H}, \mathrm{J}_{4^{\prime}, 5^{\prime}}=3.5, \mathrm{~J}_{4^{\prime} 3^{\prime}}=3.1, \mathrm{H}-4^{\prime}\right) ; 4.15$ (ddd, $\left.1 \mathrm{H}, \mathrm{J}_{3^{\prime}, 2^{\prime}}=5.1, \mathrm{~J}_{3^{\prime}, \mathrm{OH}}=4.7, \mathrm{~J}_{3^{\prime}, 4^{\prime}}=3.1, \mathrm{H}-3^{\prime}\right) ; 4.40\left(\mathrm{ddd}, 1 \mathrm{H}, \mathrm{J}_{2^{\prime}, \mathrm{OH}}=6.4, \mathrm{~J}_{2^{\prime}, 1^{\prime}}=6.3\right.$, $\left.\mathrm{J}_{2^{\prime} 3^{\prime}}=5.1, \mathrm{H}-2^{\prime}\right) ; 5.21\left(\mathrm{dd}, 1 \mathrm{H}, \mathrm{J}_{\mathrm{OH}, 5^{\prime} \mathrm{a}}=5.3, \mathrm{~J}_{\mathrm{OH}, 5^{\prime} \mathrm{b}}=5.1, \mathrm{OH}-5^{\prime}\right) ; 5.29\left(\mathrm{~d}, 1 \mathrm{H}, \mathrm{J}_{\mathrm{OH}, 3^{\prime}}=4.7\right.$, $\left.\mathrm{OH}-3^{\prime}\right) ; 5.57\left(\mathrm{~d}, 1 \mathrm{H}, \mathrm{J}_{\mathrm{OH}, 2^{\prime}}=6.4, \mathrm{OH}-2^{\prime}\right) ; 5.95\left(\mathrm{~d}, 1 \mathrm{H}, \mathrm{J}_{1^{\prime}, 2^{\prime}}=6.3, \mathrm{H}-1^{\prime}\right) ; 6.72\left(\mathrm{dd}, 1 \mathrm{H}, \mathrm{J}_{4,3}=\right.$ 3.4, $\mathrm{J}_{4,5}=1.8, \mathrm{H}$-4-furyl); $7.72\left(\mathrm{dd}, 1 \mathrm{H}, \mathrm{J}_{3,4}=3.4, \mathrm{~J}_{3,5}=0.9, \mathrm{H}\right.$-3-furyl); $7.76\left(\mathrm{~d}, 1 \mathrm{H}, \mathrm{J}_{7,6}=\right.$ 5.6, H-7); $7.90\left(\mathrm{dd}, 1 \mathrm{H}, \mathrm{J}_{5,4}=1.8, \mathrm{~J}_{5,3}=0.9, \mathrm{H}-5\right.$-furyl); $8.36\left(\mathrm{~d}, 1 \mathrm{H}, \mathrm{J}_{6,7}=5.6, \mathrm{H}-6\right) ; 8.68$ $(\mathrm{s}, 1 \mathrm{H}, \mathrm{H}-2) .{ }^{13} \mathrm{C}$ NMR (100.6 MHz, DMSO-d 6 ): $61.19\left(\mathrm{CH}_{2}-5^{\prime}\right) ; 70.18\left(\mathrm{CH}-3^{\prime}\right) ; 74.05\left(\mathrm{CH}-2^{\prime}\right)$; $85.94\left(\mathrm{CH}-4^{\prime}\right) ; 88.92\left(\mathrm{CH}-1^{\prime}\right) ; 106.24(\mathrm{CH}-7) ; 112.15$ (CH-4-furyl); 113.44 (CH-3-furyl); 136.25 (C-3a); 138.35 (C-7a); 140.24 (C-4); 141.39 (CH-6); 143.93 and 144.14 (CH-2 and CH-5-furyl); 150.81 (C-2-furyl). IR (KBr): 1596, 1499, 1480, 1407, 1311, 1216, 1121, 1107, 1084, 1013. HR MS (ESI): calculated for $\mathrm{C}_{15} \mathrm{H}_{15} \mathrm{~N}_{3} \mathrm{NaO}_{5}[\mathrm{M}+\mathrm{Na}] 340.0909$, found 340.0905 . For $\mathrm{C}_{15} \mathrm{H}_{15} \mathrm{~N}_{3} \mathrm{O}_{5} \cdot 1.5 \mathrm{H}_{2} \mathrm{O}$ calculated: $52.32 \% \mathrm{C}, 5.27 \% \mathrm{H}, 12.20 \% \mathrm{~N}$; found: $52.71 \% \mathrm{C}, 5.00 \% \mathrm{H}$, $12.03 \% \mathrm{~N}$.

1-( $\beta$-D-Ribofuranosyl)-4-(thiophen-2-yl)-1H-imidazo[4,5-c]pyridine (5c). Yield 90\%. Reaction time 3 h. Cotton-like colorless crystals from 99\% EtOH, m.p. $192-193{ }^{\circ} \mathrm{C}$. $[\alpha]_{D}-41.3$ (c 0.1 , DMSO). ${ }^{1} \mathrm{H}$ NMR $\left(400 \mathrm{MHz}, \mathrm{DMSO}-\mathrm{d}_{6}\right): 3.65\left(\mathrm{ddd}, 1 \mathrm{H}, \mathrm{J}_{\mathrm{gem}}=12.0, \mathrm{~J}_{5^{\prime} \mathrm{b}, \mathrm{OH}}=5.1, \mathrm{~J}_{5^{\prime} \mathrm{b}, 4^{\prime}}=3.5\right.$, $\left.\mathrm{H}-5^{\prime} \mathrm{b}\right)$; 3.71 (ddd, $1 \mathrm{H}$, J $\left.\mathrm{J}_{\text {gem }}=12.0, \mathrm{~J}_{5^{\prime} \mathrm{a}, \mathrm{OH}}=5.3, \mathrm{~J}_{5^{\prime} \mathrm{a}, 4^{\prime}}=3.5, \mathrm{H}-5^{\prime} \mathrm{a}\right) ; 4.03\left(\mathrm{td}, 1 \mathrm{H}, \mathrm{J}_{4^{\prime}, 5^{\prime}}=3.5\right.$, $\left.\mathrm{J}_{4^{\prime} 3^{\prime}}=3.1, \mathrm{H}-4^{\prime}\right) ; 4.15\left(\mathrm{ddd}, 1 \mathrm{H}, \mathrm{J}_{3^{\prime}, 2^{\prime}}=5.1, \mathrm{~J}_{3^{\prime}, \mathrm{OH}}=4.7, \mathrm{~J}_{3^{\prime}, 4^{\prime}}=3.1, \mathrm{H}-3^{\prime}\right) ; 4.40$ (ddd, $1 \mathrm{H}$, $\left.\mathrm{J}_{2^{\prime}, \mathrm{OH}}=6.4, \mathrm{~J}_{2^{\prime}, 1^{\prime}}=6.3, \mathrm{~J}_{2^{\prime} 3^{\prime}}=5.1, \mathrm{H}-2^{\prime}\right) ; 5.20\left(\mathrm{dd}, 1 \mathrm{H}, \mathrm{J}_{\mathrm{OH}, 5^{\prime} \mathrm{a}}=5.3, \mathrm{~J}_{\mathrm{OH}, 5^{\prime} \mathrm{b}}=5.1, \mathrm{OH}-5^{\prime}\right) ; 5.29$ $\left(\mathrm{d}, 1 \mathrm{H}, \mathrm{J}_{\mathrm{OH}, 3^{\prime}}=4.7, \mathrm{OH}-3^{\prime}\right) ; 5.57\left(\mathrm{~d}, 1 \mathrm{H}, \mathrm{J}_{\mathrm{OH}, 2^{\prime}}=6.4, \mathrm{OH}-2^{\prime}\right) ; 5.95\left(\mathrm{~d}, 1 \mathrm{H}, \mathrm{J}_{1^{\prime}, 2^{\prime}}=6.3, \mathrm{H}-1^{\prime}\right)$; $7.25\left(\mathrm{dd}, 1 \mathrm{H}, \mathrm{J}_{4,5}=5.1, \mathrm{~J}_{4,3}=3.7, \mathrm{H}\right.$-4-thienyl); $7.69\left(\mathrm{dd}, 1 \mathrm{H}, \mathrm{J}_{5,4}=5.1, \mathrm{~J}_{5,3}=1.2\right.$, $\mathrm{H}-5$-thienyl); $7.75\left(\mathrm{~d}, 1 \mathrm{H}, \mathrm{J}_{7,6}=5.6, \mathrm{H}-7\right) ; 8.31\left(\mathrm{~d}, 1 \mathrm{H}, \mathrm{J}_{6,7}=5.6, \mathrm{H}-6\right) ; 8.58\left(\mathrm{dd}, 1 \mathrm{H}, \mathrm{J}_{3,4}=\right.$ 3.7, $\mathrm{J}_{3,5}=1.2, \mathrm{H}-3$-thienyl); $8.71(\mathrm{~s}, 1 \mathrm{H}, \mathrm{H}-2) .{ }^{13} \mathrm{C} \mathrm{NMR}(100.6 \mathrm{MHz}$ DMSO-d 6 ): 61.16 $\left(\mathrm{CH}_{2}-5^{\prime}\right) ; 70.15\left(\mathrm{CH}-3^{\prime}\right) ; 74.04\left(\mathrm{CH}-2^{\prime}\right) ; 85.93\left(\mathrm{CH}-4^{\prime}\right) ; 88.93\left(\mathrm{CH}-1^{\prime}\right) ; 106.19(\mathrm{CH}-7) ; 128.37$ (CH-4-thienyl); 128.46 (CH-5-thienyl); 129.06 (CH-3-thienyl); 136.32 (C-3a); 138.53 (C-7a); 141.34 (CH-6); 142.53 (C-2-thienyl); 143.66 (C-4); 144.00 (CH-2). IR (KBr): 1592, 1578, 1465, 1278, 1213, 1131, 1085, 1075, 1065, 1046, 992. HR MS (ESI): calculated for 
$\mathrm{C}_{15} \mathrm{H}_{16} \mathrm{~N}_{3} \mathrm{O}_{4} \mathrm{~S}[\mathrm{M}+\mathrm{H}]$ 334.0862, found 334.0859. For $\mathrm{C}_{15} \mathrm{H}_{15} \mathrm{~N}_{3} \mathrm{O}_{4} \mathrm{~S}$ calculated: $54.04 \% \mathrm{C}$, $4.54 \% \mathrm{H}, 12.60 \% \mathrm{~N}, 9.62 \% \mathrm{~S}$; found: $53.74 \% \mathrm{C}, 4.48 \% \mathrm{H}, 12.45 \% \mathrm{~N}, 9.46 \% \mathrm{~S}$.

4-(Furan-3-yl)-1-( $\beta$-D-ribofuranosyl)-1H-imidazo[4,5-c]pyridine (5d). Yield 72\%. Reaction time 2 h. Cotton-like white crystals from water, m.p. $181-186{ }^{\circ} \mathrm{C} .[\alpha]_{D}-59.3$ (c 1.8, DMSO). ${ }^{1} \mathrm{H}$ NMR (400 MHz, DMSO-d ${ }_{6}$ ): 3.65 (ddd, $\left.1 \mathrm{H}, \mathrm{J}_{\text {gem }}=12.0, \mathrm{~J}_{5^{\prime} \mathrm{b}, \mathrm{OH}}=5.1, \mathrm{~J}_{5^{\prime} \mathrm{b}, 4^{\prime}}=3.5, \mathrm{H}-5^{\prime} \mathrm{b}\right)$; $3.70\left(\mathrm{ddd}, 1 \mathrm{H}, \mathrm{J}_{\mathrm{gem}}=12.0, \mathrm{~J}_{5^{\prime} \mathrm{a}, \mathrm{OH}}=5.3, \mathrm{~J}_{5^{\prime} \mathrm{a}, 4^{\prime}}=3.5, \mathrm{H}-5^{\prime} \mathrm{a}\right) ; 4.04\left(\mathrm{td}, 1 \mathrm{H}, \mathrm{J}_{4^{\prime}, 5^{\prime}}=3.5, \mathrm{~J}_{4^{\prime}, 3^{\prime}}=\right.$ $\left.3.1, \mathrm{H}-4^{\prime}\right) ; 4.15\left(\mathrm{ddd}, 1 \mathrm{H}, \mathrm{J}_{3^{\prime}, 2^{\prime}}=5.1, \mathrm{~J}_{3^{\prime}, \mathrm{OH}}=4.7, \mathrm{~J}_{3^{\prime}, 4^{\prime}}=3.1, \mathrm{H}-3^{\prime}\right) ; 4.39\left(\mathrm{ddd}, 1 \mathrm{H}, \mathrm{J}_{2^{\prime}, \mathrm{OH}}=\right.$ $\left.6.4, \mathrm{~J}_{2^{\prime}, 1^{\prime}}=6.3, \mathrm{~J}_{2^{\prime}, 3^{\prime}}=5.1, \mathrm{H}-2^{\prime}\right) ; 5.19\left(\mathrm{dd}, 1 \mathrm{H}, \mathrm{J}_{\mathrm{OH}, 5^{\prime} \mathrm{a}}=5.3, \mathrm{~J}_{\mathrm{OH}, 5^{\prime} \mathrm{b}}=5.1, \mathrm{OH}-5^{\prime}\right) ; 5.28(\mathrm{~d}$, $\left.1 \mathrm{H}, \mathrm{J}_{\mathrm{OH}, 3^{\prime}}=4.7, \mathrm{OH}-3^{\prime}\right) ; 5.55\left(\mathrm{~d}, 1 \mathrm{H}, \mathrm{J}_{\mathrm{OH}, 2^{\prime}}=6.4, \mathrm{OH}-2^{\prime}\right) ; 5.94\left(\mathrm{~d}, 1 \mathrm{H}, \mathrm{J}_{1^{\prime}, 2^{\prime}}=6.3, \mathrm{H}-1^{\prime}\right) ; 7.33$ (dd, $1 \mathrm{H}, \mathrm{J}_{4,5}=1.8, \mathrm{~J}_{4,2}=0.8, \mathrm{H}$-4-furyl); $7.73\left(\mathrm{~d}, 1 \mathrm{H}, \mathrm{J}_{7,6}=5.5, \mathrm{H}-7\right) ; 7.82\left(\mathrm{dd}, 1 \mathrm{H}, \mathrm{J}_{5,4}=\right.$ 1.8, J $\mathrm{J}_{5,2}=1.6, \mathrm{H}-5$-furyl); $8.35\left(\mathrm{~d}, 1 \mathrm{H}, \mathrm{J}_{6,7}=5.6, \mathrm{H}-6\right) ; 8.66(\mathrm{~s}, 1 \mathrm{H}, \mathrm{H}-2) ; 8.75\left(\mathrm{dd}, 1 \mathrm{H}, \mathrm{J}_{2,5}=\right.$ 1.6, $\mathrm{J}_{2,4}=0.8, \mathrm{H}-2$-furyl). ${ }^{13} \mathrm{C} \mathrm{NMR}\left(100.6 \mathrm{MHz}, \mathrm{DMSO}-\mathrm{d}_{6}\right): 61.17\left(\mathrm{CH}_{2}-5^{\prime}\right) ; 70.16\left(\mathrm{CH}-3^{\prime}\right)$; 74.00 (CH-2'); 85.89 (CH-4'); $88.89\left(\mathrm{CH}^{\prime} \mathbf{1}^{\prime}\right) ; 105.85$ (CH-7); 109.40 (CH-4-furyl); 124.62 (C-3-furyl); 137.43 (C-3a); 138.01 (C-7a); 141.47 (CH-6); 143.08 (C-4); 143.60 (2 C, CH-2 and CH-5-furyl); 143.82 (CH-2-furyl). IR (KBr): 1592, 1304, 1222, 1111, 1096, 1066, 873. HR MS (ESI): calculated for $\mathrm{C}_{15} \mathrm{H}_{16} \mathrm{~N}_{3} \mathrm{O}_{5}[\mathrm{M}+\mathrm{H}]$ 318.1090, found 318.1088. For $\mathrm{C}_{15} \mathrm{H}_{15} \mathrm{~N}_{3} \mathrm{O}_{5} \cdot \mathrm{H}_{2} \mathrm{O}$ calculated: $53.73 \% \mathrm{C}, 5.11 \% \mathrm{H}, 12.53 \% \mathrm{~N}$; found: $53.74 \% \mathrm{C}, 4.96 \% \mathrm{H}, 12.50 \% \mathrm{~N}$.

1-( $\beta$-D-Ribofuranosyl)-4-(thiophen-3-yl)-1H-imidazo[4,5-c]pyridine (5e). Yield 78\%. Reaction time $2 \mathrm{~h}$. Yellowish crystals from water, m.p. $105-109^{\circ} \mathrm{C}$. $[\alpha]_{D}-66.5$ (c 2.6, DMSO). ${ }^{1} \mathrm{H}$ NMR $\left(400 \mathrm{MHz}, \mathrm{DMSO}-\mathrm{d}_{6}\right.$ ): 3.65 (ddd, $1 \mathrm{H}, \mathrm{J}_{\mathrm{gem}}=12.0 \mathrm{~J}_{5^{\prime} \mathrm{b}, \mathrm{OH}}=5.1, \mathrm{~J}_{5^{\prime} \mathrm{b}, 4^{\prime}}=3.5, \mathrm{H}-5^{\prime} \mathrm{b}$ ); 3.71 (ddd, $\left.1 \mathrm{H}, \mathrm{J}_{\mathrm{gem}}=12.0, \mathrm{~J}_{5^{\prime} \mathrm{a}, \mathrm{OH}}=5.4, \mathrm{~J}_{5^{\prime} \mathrm{a}, 4^{\prime}}=3.5, \mathrm{H}-5^{\prime} \mathrm{a}\right) ; 4.03\left(\mathrm{td}, 1 \mathrm{H}, \mathrm{J}_{4^{\prime}, 5^{\prime}}=3.5, \mathrm{~J}_{4^{\prime} 3^{\prime}}=3.1, \mathrm{H}-4^{\prime}\right)$; $4.16\left(\mathrm{ddd}, 1 \mathrm{H}, \mathrm{J}_{3^{\prime}, 2^{\prime}}=5.1, \mathrm{~J}_{3^{\prime}, \mathrm{OH}}=4.7, \mathrm{~J}_{3^{\prime}, 4^{\prime}}=3.1, \mathrm{H}-3^{\prime}\right) ; 4.41\left(\mathrm{ddd}, 1 \mathrm{H}, \mathrm{J}_{2^{\prime}, \mathrm{OH}}=6.4, \mathrm{~J}_{2^{\prime}, 1^{\prime}}=\right.$ $\left.6.3, \mathrm{~J}_{2^{\prime} 3^{\prime}}=5.1, \mathrm{H}-2^{\prime}\right) ; 5.20\left(\mathrm{dd}, 1 \mathrm{H}, \mathrm{J}_{\mathrm{OH}, 5^{\prime} \mathrm{a}}=5.4, \mathrm{~J}_{\mathrm{OH}, 5^{\prime} \mathrm{b}}=5.1, \mathrm{OH}-5^{\prime}\right) ; 5.29\left(\mathrm{~d}, 1 \mathrm{H}, \mathrm{J}_{\mathrm{OH}, 3^{\prime}}=\right.$ 4.7, $\left.\mathrm{OH}-3^{\prime}\right) ; 5.56\left(\mathrm{~d}, 1 \mathrm{H}, \mathrm{J}_{\mathrm{OH}, 2^{\prime}}=6.4, \mathrm{OH}-2^{\prime}\right) ; 5.95\left(\mathrm{~d}, 1 \mathrm{H}, \mathrm{J}_{1^{\prime}, 2^{\prime}}=6.3, \mathrm{H}-\mathrm{I}^{\prime}\right) ; 7.65$ (dd, $1 \mathrm{H}$, $\mathrm{J}_{5,4}=5.1, \mathrm{~J}_{5,2}=3.0, \mathrm{H}-5$-thienyl); $7.76\left(\mathrm{~d}, 1 \mathrm{H}, \mathrm{J}_{7,6}=5.6, \mathrm{H}-7\right) ; 8.21\left(\mathrm{dd}, 1 \mathrm{H}, \mathrm{J}_{4,5}=5.1, \mathrm{~J}_{4,2}=\right.$ 1.1, H-4-thienyl); $8.38\left(\mathrm{~d}, 1 \mathrm{H}, \mathrm{J}_{6,7}=5.6, \mathrm{H}-6\right) ; 8.70(\mathrm{~s}, 1 \mathrm{H}, \mathrm{H}-2) ; 8.83\left(\mathrm{dd}, 1 \mathrm{H}, \mathrm{J}_{2,5}=3.0\right.$, $\mathrm{J}_{2,4}=1.1, \mathrm{H}$-2-thienyl). ${ }^{13} \mathrm{C}$ NMR $\left(100.6 \mathrm{MHz}, \mathrm{DMSO}-\mathrm{d}_{6}\right): 61.18\left(\mathrm{CH}_{2}-5^{\prime}\right) ; 70.16\left(\mathrm{CH}-3^{\prime}\right)$; $74.00\left(\mathrm{CH}-2^{\prime}\right) ; 85.89\left(\mathrm{CH}-4^{\prime}\right) ; 88.89\left(\mathrm{CH}-\mathrm{I}^{\prime}\right) ; 106.07(\mathrm{CH}-7) ; 125.80$ (CH-5-thienyl); 126.89 (CH-2-thienyl); 127.63 (CH-4-thienyl); 137.44 (C-3a); 138.58 (C-7a); 140.01 (C-3-thienyl); 141.39 (CH-6); 143.71 (CH-2); 144.50 (C-4). IR (KBr): 1596, 1580, 1463, 1221, 1106, 1061, 1037. HR MS (ESI): calculated for $\mathrm{C}_{15} \mathrm{H}_{16} \mathrm{~N}_{3} \mathrm{O}_{4} \mathrm{~S}[\mathrm{M}+\mathrm{H}]$ 334.0862, found 334.0857. For $\mathrm{C}_{15} \mathrm{H}_{15} \mathrm{~N}_{3} \mathrm{O}_{4} \mathrm{~S} \cdot \mathrm{H}_{2} \mathrm{O}$ calculated: $51.27 \% \mathrm{C}, 4.88 \% \mathrm{H}, 11.96 \% \mathrm{~N}$; found: $51.13 \% \mathrm{C}, 4.81 \% \mathrm{H}$, $11.75 \% \mathrm{~N}$.

4-(Pyrrol-2-yl)-1-( $\beta$-D-ribofuranosyl)-1H-imidazo[4,5-c]pyridine (5f). Yield 50\%. Reaction time 24 h. White crystals from MeOH, m.p. 205-206 ${ }^{\circ} \mathrm{C}$. [ $[\alpha]_{\mathrm{D}}-53.4$ (c 1.9, DMSO). ${ }^{1} \mathrm{H}$ NMR $\left(400 \mathrm{MHz}, \mathrm{DMSO}-\mathrm{d}_{6}\right)$ : $3.64\left(\mathrm{ddd}, 1 \mathrm{H}, \mathrm{J}_{\mathrm{gem}}=12.0, \mathrm{~J}_{5^{\prime} \mathrm{b}, \mathrm{OH}}=5.1, \mathrm{~J}_{5^{\prime} \mathrm{b}, 4^{\prime}}=3.5, \mathrm{H}-5^{\prime} \mathrm{b}\right)$ ); 3.70 (ddd, $\left.1 \mathrm{H}, \mathrm{J}_{\text {gem }}=12.0, \mathrm{~J}_{5^{\prime} \mathrm{a}, \mathrm{OH}}=5.3, \mathrm{~J}_{5^{\prime} \mathrm{a}, 4^{\prime}}=3.5, \mathrm{H}-5^{\prime} \mathrm{a}\right) ; 4.01\left(\mathrm{td}, 1 \mathrm{H}, \mathrm{J}_{4^{\prime}, 5^{\prime}}=3.5, \mathrm{~J}_{4^{\prime}, 3^{\prime}}=3.0, \mathrm{H}-4^{\prime}\right)$; 4.14 (ddd, $\left.1 \mathrm{H}, \mathrm{J}_{3^{\prime}, 2^{\prime}}=5.1, \mathrm{~J}_{3^{\prime}, \mathrm{OH}}=4.6, \mathrm{~J}_{3^{\prime}, 4^{\prime}}=3.0, \mathrm{H}-3^{\prime}\right) ; 4.39\left(\mathrm{ddd}, 1 \mathrm{H}, \mathrm{J}_{2^{\prime}, \mathrm{OH}}=6.1, \mathrm{~J}_{2^{\prime}, 1^{\prime}}=\right.$ $\left.6.3, \mathrm{~J}_{2^{\prime}, 3^{\prime}}=5.1, \mathrm{H}-2^{\prime}\right) ; 5.18\left(\mathrm{dd}, 1 \mathrm{H}, \mathrm{J}_{\mathrm{OH}, 5^{\prime} \mathrm{a}}=5.3, \mathrm{~J}_{\mathrm{OH}, 5^{\prime} \mathrm{b}}=5.1, \mathrm{OH}-5^{\prime}\right) ; 5.28\left(\mathrm{~d}, 1 \mathrm{H}, \mathrm{J}_{\mathrm{OH}, 3^{\prime}}=\right.$ 4.6, $\left.\mathrm{OH}-3^{\prime}\right) ; 5.56\left(\mathrm{~d}, 1 \mathrm{H}, \mathrm{J}_{\mathrm{OH}, 2^{\prime}}=6.1, \mathrm{OH}-2^{\prime}\right) ; 5.91\left(\mathrm{~d}, 1 \mathrm{H}, \mathrm{J}_{1^{\prime}, 2^{\prime}}=6.3, \mathrm{H}-1^{\prime}\right) ; 6.21(\mathrm{dt}, 1 \mathrm{H}$, $\mathrm{J}_{4,3}=3.7, \mathrm{~J}_{4,5}=\mathrm{J}_{4, \mathrm{NH}}=2.4, \mathrm{H}-4$-pyrr); $6.95\left(\mathrm{ddd}, 1 \mathrm{H}, \mathrm{J}_{5, \mathrm{NH}}=2.7, \mathrm{~J}_{5,4}=2.4, \mathrm{~J}_{5,3}=1.6\right.$, $\mathrm{H}$-5-pyrr); 7.38 (ddd, $\left.1 \mathrm{H}, \mathrm{J}_{3,4}=3.7, \mathrm{~J}_{3, \mathrm{NH}}=2.3, \mathrm{~J}_{3,5}=1.6, \mathrm{H}-3-p y r r\right) ; 7.58\left(\mathrm{~d}, 1 \mathrm{H}, \mathrm{J}_{7,6}=5.6\right.$, $\mathrm{H}-7) ; 8.26\left(\mathrm{~d}, 1 \mathrm{H}, \mathrm{J}_{6,7}=5.6, \mathrm{H}-6\right) ; 8.63(\mathrm{~s}, 1 \mathrm{H}, \mathrm{H}-2) ; 11.54$ (br s, $\left.1 \mathrm{H}, \mathrm{NH}-\mathrm{pyrr}\right) .{ }^{13} \mathrm{C}$ NMR $\left(100.6 \mathrm{MHz}\right.$ DMSO-d 6 ): $61.18\left(\mathrm{CH}_{2}-5^{\prime}\right) ; 70.14\left(\mathrm{CH}-3^{\prime}\right) ; 73.97\left(\mathrm{CH}-2^{\prime}\right) ; 85.81\left(\mathrm{CH}-4^{\prime}\right) ; 88.80$ (CH-1'); 104.27 (CH-7); 109.28 (CH-4-pyrr); 111.56 (CH-3-pyrr); 120.89 (CH-5-pyrr); 129.58 (C-2-pyrr); 135.53 (C-3a); 138.17 (C-7a); 141.17 (CH-6); 142.71 (C-4); 143.08 (CH-2). IR (KBr): 3352, 1601, 1588, 1470, 1220, 1135, 1084, 1063, 1045. HR MS (ESI): calculated for 
$\mathrm{C}_{15} \mathrm{H}_{17} \mathrm{~N}_{4} \mathrm{O}_{4}[\mathrm{M}+\mathrm{H}]$ 317.1250, found 317.1248. For $\mathrm{C}_{15} \mathrm{H}_{16} \mathrm{~N}_{4} \mathrm{O}_{4} \cdot 0.5 \mathrm{H}_{2} \mathrm{O}$ calculated: $55.38 \% \mathrm{C}$, $5.27 \% \mathrm{H}, 17.22 \% \mathrm{~N}$; found: $55.49 \% \mathrm{C}, 4.92 \% \mathrm{H}, 16.95 \% \mathrm{~N}$.

4-(Pyrrol-3-yl)-1-( $\beta$-D-ribofuranosyl)-1H-imidazo[4,5-c]pyridine (5g). Yield 61\%. Reaction time 15 h. Greenish solid from water, m.p. $119-126{ }^{\circ} \mathrm{C}$. $[\alpha]_{\mathrm{D}}-48.8$ (c 1.7, DMSO). ${ }^{1} \mathrm{H}$ NMR (400 MHz, DMSO- $d_{6}$ ): $3.64\left(d d d, 1 \mathrm{H}\right.$, J gem $\left.=11.9, \mathrm{~J}_{5^{\prime} \mathrm{b}, \mathrm{OH}}=5.1, \mathrm{~J}_{5^{\prime} \mathrm{b}, 4^{\prime}}=3.65, \mathrm{H}-5^{\prime} \mathrm{b}\right) ; 3.70\left(\mathrm{ddd}, 1 \mathrm{H}\right.$, J $\mathrm{Jem}_{\text {gem }}=$ $\left.11.9, \mathrm{~J}_{5^{\prime} \mathrm{a}, \mathrm{OH}}=5.3, \mathrm{~J}_{5^{\prime} \mathrm{a}, 4^{\prime}}=3.6, \mathrm{H}-5^{\prime} \mathrm{a}\right) ; 4.00\left(\mathrm{td}, 1 \mathrm{H}, \mathrm{J}_{4^{\prime}, 5^{\prime}}=3.6, \mathrm{~J}_{4^{\prime} 3^{\prime}}=3.2, \mathrm{H}-4^{\prime}\right) ; 4.14$ (ddd, $1 \mathrm{H}$, $\left.\mathrm{J}_{3^{\prime}, 2^{\prime}}=5.1, \mathrm{~J}_{3^{\prime}, \mathrm{OH}}=4.7, \mathrm{~J}_{3^{\prime}, 4^{\prime}}=3.2, \mathrm{H}-3^{\prime}\right) 4.39\left(\mathrm{ddd}, 1 \mathrm{H}, \mathrm{J}_{2^{\prime}, \mathrm{OH}}=6.5, \mathrm{~J}_{2^{\prime}, 1^{\prime}}=6.3, \mathrm{~J}_{2^{\prime} 3^{\prime}}=5.1\right.$, $\left.\mathrm{H}-2^{\prime}\right) ; 5.17\left(\mathrm{dd}, 1 \mathrm{H}, \mathrm{J}_{\mathrm{OH}, 5^{\prime} \mathrm{a}}=5.3, \mathrm{~J}_{\mathrm{OH}, 5^{\prime} \mathrm{b}}=5.1, \mathrm{OH}-5^{\prime}\right) ; 5.26\left(\mathrm{~d}, 1 \mathrm{H}, \mathrm{J}_{\mathrm{OH}, 3^{\prime}}=4.7, \mathrm{OH}-3^{\prime}\right) ; 5.54$ $\left(\mathrm{d}, 1 \mathrm{H}, \mathrm{J}_{\mathrm{OH}, 2^{\prime}}=6.5, \mathrm{OH}-2^{\prime}\right) ; 5.89\left(\mathrm{~d}, 1 \mathrm{H}, \mathrm{J}_{1^{\prime}, 2^{\prime}}=6.3, \mathrm{H}-\mathrm{I}^{\prime}\right) ; 6.84\left(\mathrm{td}, 1 \mathrm{H}, \mathrm{J}_{5,4}=\mathrm{J}_{5, \mathrm{NH}}=2.6\right.$, $\mathrm{J}_{5,2}=2.0, \mathrm{H}-5$-pyrr); $7.03\left(\mathrm{td}, 1 \mathrm{H}, \mathrm{J}_{4,5}=\mathrm{J}_{4, \mathrm{NH}}=2.6, \mathrm{~J}_{4,2}=1.5, \mathrm{H}-4\right.$-pyrr); $7.51\left(\mathrm{~d}, 1 \mathrm{H}, \mathrm{J}_{7,6}=\right.$ 5.6, H-7); 8.03 (ddd, $1 \mathrm{H}, \mathrm{J}_{2, \mathrm{NH}}=2.8, \mathrm{~J}_{2,5}=2.0, \mathrm{~J}_{2,4}=1.5, \mathrm{H}-2$-pyrr); $8.23\left(\mathrm{~d}, 1 \mathrm{H}, \mathrm{J}_{6,7}=5.6\right.$, $\mathrm{H}-6) ; 8.55$ (s, $1 \mathrm{H}, \mathrm{H}-2$ ); 11.10 (br s, $1 \mathrm{H}, \mathrm{NH}-\mathrm{pyrr}) .{ }^{13} \mathrm{C} \mathrm{NMR}(100.6 \mathrm{MHz}$, DMSO-d 6 ): 61.23 $\left(\mathrm{CH}_{2}-5^{\prime}\right) ; 70.16\left(\mathrm{CH}-3^{\prime}\right) ; 73.90\left(\mathrm{CH}-2^{\prime}\right) ; 85.72\left(\mathrm{CH}-4^{\prime}\right) ; 88.72\left(\mathrm{CH}-1^{\prime}\right) ; 103.68(\mathrm{CH}-7) ; 107.69$ (CH-4-pyrr); 118.23 (CH-5-pyrr); 120.58 (CH-2-pyrr); 122.08 (C-3-pyrr); 136.47 (C-3a); 137.94 (C-7a); 141.26 (CH-6); 142.47 (CH-2); 146.96 (C-4). IR (KBr): 3388, 1588, 1552, 1480, 1460, 1217, 1111, 1063, 1041. HR MS (ESI): calculated for $\mathrm{C}_{15} \mathrm{H}_{17} \mathrm{~N}_{4} \mathrm{O}_{4}[\mathrm{M}+\mathrm{H}] 317.1250$, found 317.1245. For $\mathrm{C}_{15} \mathrm{H}_{16} \mathrm{~N}_{4} \mathrm{O}_{4} \cdot 1.5 \mathrm{H}_{2} \mathrm{O}$ calculated: $52.47 \% \mathrm{C}, 5.58 \% \mathrm{H}, 16.32 \% \mathrm{~N}$; found: $52.76 \% \mathrm{C}, 5.45 \% \mathrm{H}, 16.04 \% \mathrm{~N}$.

4-(1H-Pyrazol-5-yl)-1-( $\beta$-D-ribofuranosyl)- $1 \mathrm{H}$-imidazo[4,5-c]pyridine (5h). Yield $10 \%$ after crystalization, $82 \%$ of starting compound 4 recovered. Reaction time $24 \mathrm{~h}$. Colorless crystals from water, m.p. ${ }^{139-143}{ }^{\circ} \mathrm{C}$. $[\alpha]_{D}-59.0$ (c 1.7, DMSO). ${ }^{1} \mathrm{H}$ NMR (500 M Hz, DMSO-d 6 + DCl): 3.66, $3.71\left(2 \times \mathrm{dd}, 2 \times 1 \mathrm{H}, \mathrm{J}_{\mathrm{gem}}=12.2, \mathrm{~J}_{5^{\prime}, 4^{\prime}}=3.2, \mathrm{H}-5^{\prime} \mathrm{b}\right) ; 4.08\left(\mathrm{dt}, 1 \mathrm{H}, \mathrm{J}_{4^{\prime}, 3^{\prime}}=3.6, \mathrm{~J}_{4^{\prime}, 5^{\prime}}=\right.$ $\left.3.2, \mathrm{H}-4^{\prime}\right) ; 4.17\left(\mathrm{dd}, 1 \mathrm{H}, \mathrm{J}_{3^{\prime}, 2^{\prime}}=5.1, \mathrm{~J}_{3^{\prime}, 4^{\prime}}=3.6, \mathrm{H}-3^{\prime}\right) ; 4.37\left(\mathrm{dd}, 1 \mathrm{H}, \mathrm{J}_{2^{\prime}, 1^{\prime}}=5.7, \mathrm{~J}_{2^{\prime}, 3^{\prime}}=5.1\right.$, $\left.\mathrm{H}-2^{\prime}\right) ; 6.14\left(\mathrm{~d}, 1 \mathrm{H}, \mathrm{J}_{1^{\prime}, 2^{\prime}}=5.7, \mathrm{H}-1^{\prime}\right) ; 7.64\left(\mathrm{~d}, 1 \mathrm{H}, \mathrm{J}_{4,5}=2.4, \mathrm{H}-4\right.$-pyrazolyl); $8.13(\mathrm{~d}, 1 \mathrm{H}$, $\mathrm{J}_{5,4}=2.4, \mathrm{H}-5$-pyrazolyl); $8.38\left(\mathrm{~d}, 1 \mathrm{H}, \mathrm{J}_{7,6}=6.7, \mathrm{H}-7\right) ; 8.48\left(\mathrm{~d}, 1 \mathrm{H}, \mathrm{J}_{6,7}=6.7, \mathrm{H}-6\right) ; 9.17$ (s, $1 \mathrm{H}, \mathrm{H}-2) .{ }^{13} \mathrm{C}$ NMR (125.7 MHz, DMSO-d 6 + DCl): $60.97\left(\mathrm{CH}_{2}-5^{\prime}\right) ; 70.25\left(\mathrm{CH}-3^{\prime}\right) ; 75.19$ $\left(\mathrm{CH}-2^{\prime}\right) ; 86.85$ ( $\left.\mathrm{CH}-4^{\prime}\right) ; 90.20\left(\mathrm{CH}-1^{\prime}\right) ; 108.95$ (CH-7); 109.25 (CH-4-pyrazolyl); 132.09 (CH-5-pyrazolyl); 134.12 (CH-6); 137.18 (C-3a); 138.05 (C-4); 140.54 (C-3-pyrazolyl); 143.30 (C-7a); 148.84 (CH-2). IR (KBr): 3429, 1633, 1605, 1592, 1482, 1402, 1304, 1221, 1137 , 1112, 1084. HR MS (ESI): calculated for $\mathrm{C}_{14} \mathrm{H}_{15} \mathrm{~N}_{5} \mathrm{NaO}_{4}[\mathrm{M}+\mathrm{Na}] 340.1022$, found 340.1018. For $\mathrm{C}_{14} \mathrm{H}_{15} \mathrm{~N}_{5} \mathrm{O}_{4} \cdot 1.35 \mathrm{H}_{2} \mathrm{O}$ calculated: $49.22 \% \mathrm{C}, 5.22 \% \mathrm{H}, 20.50 \% \mathrm{~N}$; found: $49.47 \% \mathrm{C}$, $5.09 \% \mathrm{H}, 20.22 \% \mathrm{~N}$.

4-(Pyridin-3-yl)-1-( $\beta$-D-ribofuranosyl)-1H-imidazo[4,5-c]pyridine (5i). Yield 96\%. Reaction time 20 h. White crystalline solid from water, m.p. $111-115^{\circ} \mathrm{C}$. $[\alpha]_{D}-55.3$ (c 1.7, DMSO). ${ }^{1} \mathrm{H}$ NMR $\left(400 \mathrm{MHz}, \mathrm{DMSO}-\mathrm{d}_{6}\right)$ : 3.66 (ddd, $1 \mathrm{H}, \mathrm{J}_{\mathrm{gem}}=12.0$, J $_{5^{\prime} \mathrm{b}, \mathrm{OH}}=5.1, \mathrm{~J}_{5^{\prime} \mathrm{b}, 4^{\prime}}=3.5, \mathrm{H}-5^{\prime} \mathrm{b}$ ); 3.72 (ddd, $\left.1 \mathrm{H}, \mathrm{J}_{\mathrm{gem}}=12.0, \mathrm{~J}_{5^{\prime} \mathrm{a}, \mathrm{OH}}=5.3, \mathrm{~J}_{5^{\prime} \mathrm{a}, 4^{\prime}}=3.5, \mathrm{H}-5^{\prime} \mathrm{a}\right) ; 4.04\left(\mathrm{td}, 1 \mathrm{H}, \mathrm{J}_{4^{\prime}, 5^{\prime}}=3.5, \mathrm{~J}_{4^{\prime}, 3^{\prime}}=3.1, \mathrm{H}-4^{\prime}\right)$; $4.16\left(\mathrm{ddd}, 1 \mathrm{H}, \mathrm{J}_{3^{\prime}, 2^{\prime}}=5.1, \mathrm{~J}_{3^{\prime}, \mathrm{OH}}=4.7, \mathrm{~J}_{3^{\prime}, 4^{\prime}}=3.1, \mathrm{H}-3^{\prime}\right) ; 4.42\left(\mathrm{ddd}, 1 \mathrm{H}, \mathrm{J}_{2^{\prime}, \mathrm{OH}}=6.3, \mathrm{~J}_{2^{\prime}, 1^{\prime}}=\right.$ $\left.6.3, \mathrm{~J}_{2^{\prime}, 3^{\prime}}=5.1, \mathrm{H}-2^{\prime}\right) ; 5.22\left(\mathrm{dd}, 1 \mathrm{H}, \mathrm{J}_{\mathrm{OH}, 5^{\prime} \mathrm{a}}=5.3\right.$, $\left.\mathrm{J}_{\mathrm{OH}, 5^{\prime} \mathrm{b}}=5.1, \mathrm{OH}-5^{\prime}\right) ; 5.31\left(\mathrm{~d}, 1 \mathrm{H}, \mathrm{J}_{\mathrm{OH}, 3^{\prime}}=\right.$ 4.7, OH-3'); $5.59\left(\mathrm{~d}, 1 \mathrm{H}, \mathrm{J}_{\mathrm{OH}, 2^{\prime}}=6.3, \mathrm{OH}-2^{\prime}\right) ; 5.99\left(\mathrm{~d}, 1 \mathrm{H}, \mathrm{J}_{1^{\prime}, 2^{\prime}}=6.3, \mathrm{H}-\mathrm{I}^{\prime}\right) ; 7.57$ (ddd, $1 \mathrm{H}$, $\left.\mathrm{J}_{5,4}=8.0, \mathrm{~J}_{5,6}=4.8, \mathrm{~J}_{5,2}=1.0, \mathrm{H}-5-\mathrm{py}\right) ; 7.90\left(\mathrm{~d}, 1 \mathrm{H}, \mathrm{J}_{7,6}=5.5, \mathrm{H}-7\right) ; 8.50\left(\mathrm{~d}, 1 \mathrm{H}, \mathrm{J}_{6,7}=5.5\right.$, $\mathrm{H}-6$ ); 8.65 (dd, $\left.1 \mathrm{H}, \mathrm{J}_{6,5}=4.7, \mathrm{~J}_{6,4}=1.7, \mathrm{H}-6-\mathrm{py}\right) ; 8.75$ (s, $1 \mathrm{H}, \mathrm{H}-2$ ); 8.97 (ddd, $1 \mathrm{H}, \mathrm{J}_{4,5}=8.0$, $\left.\mathrm{J}_{4,2}=2.2, \mathrm{~J}_{4,6}=1.7, \mathrm{H}-4-\mathrm{py}\right) ; 9.81$ (dd, $\left.1 \mathrm{H}, \mathrm{J}_{2,4}=2.2, \mathrm{~J}_{2,5}=1.0, \mathrm{H}-2-\mathrm{py}\right) .{ }^{13} \mathrm{C} \mathrm{NMR}(100.6 \mathrm{MHz}$, DMSO-d 6 ): $61.17\left(\mathrm{CH}_{2}-5^{\prime}\right) ; 70.18\left(\mathrm{CH}-3^{\prime}\right) ; 74.06\left(\mathrm{CH}-2^{\prime}\right) ; 85.99\left(\mathrm{CH}-4^{\prime}\right) ; 88.97\left(\mathrm{CH}-\mathrm{I}^{\prime}\right) ; 107.20$ (CH-7); 123.53 (CH-5-py); 133.14 (C-3-py); 135.89 (CH-4-py); 138.51 (C-3a); 138.97 (C-7a); 141.74 (CH-6); 144.21 (CH-2); 145.37 (C-4); 149.69 and 149.78 (CH-2,6-py). IR (KBr): 1606, 1592, 1583, 1502, 1460, 1101, 1050, 984, 940. HR MS (ESI): calculated for $\mathrm{C}_{16} \mathrm{H}_{17} \mathrm{~N}_{4} \mathrm{O}_{4}$ 
[M + H] 329.1250, found 329.1247. For $\left.\mathrm{C}_{16} \mathrm{H}_{16} \mathrm{~N}_{4} \mathrm{O}_{4}\right) \cdot 1.8 \mathrm{H}_{2} \mathrm{O}$ calculated: $53.27 \% \mathrm{C}, 5.48 \% \mathrm{H}$, $15.53 \% \mathrm{~N}$; found: $53.42 \% \mathrm{C}, 5.37 \% \mathrm{H}, 15.34 \% \mathrm{~N}$.

4-M ethyl-1-(2,3,5-tri-0-acetyl- $\beta$-D-ribofuranosyl)-1H-imidazo[4,5-c]pyridine (6a)

An argon purged flask containing a mixture of acetylated 6-chloro-3-deazapurine riboside 3 (300 mg, $0.73 \mathrm{mmol}$ ), trimethylaluminum ( $2 \mathrm{M}$ solution in toluene; $0.73 \mathrm{ml}, 1.46 \mathrm{mmol}$ ) and $\mathrm{Pd}\left(\mathrm{PPh}_{3}\right)_{4}(42 \mathrm{mg}, 0.036 \mathrm{mmol})$ in $\operatorname{THF}(4 \mathrm{ml})$ was stirred at $100{ }^{\circ} \mathrm{C}$ for $3 \mathrm{~h}$. The mixture was diluted with $\mathrm{CHCl}_{3}(20 \mathrm{ml})$ and treated with saturated aqueous $\mathrm{NH}_{4} \mathrm{Cl}(20 \mathrm{ml})$. The slurry was filtered through cellite and after phase separation, aqueous phase was re-extracted with $\mathrm{CHCl}_{3}(2 \times 10 \mathrm{ml})$. Collected organic extracts were dried over $\mathrm{MgSO}_{4}$, volatiles were removed in vacuo and the residue was chromatographed on silica gel (AcOEt) affording product $6 \mathbf{a}$ as colorless oil (151 mg, 53\%). ${ }^{1} \mathrm{H}$ NMR (400 M Hz, CDCl $): 2.09,2.17$ and $2.19(3 \times \mathrm{s}$, $\left.3 \times 3 \mathrm{H}, 3 \times \mathrm{CH}_{3} \mathrm{CO}\right) ; 2.90\left(\mathrm{~s}, 3 \mathrm{H}, \mathrm{CH}_{3}\right) ; 4.41\left(\mathrm{dd}, 1 \mathrm{H}, \mathrm{J}_{\text {gem }}=12.6, \mathrm{~J}_{5^{\prime} \mathrm{b}, 4^{\prime}}=2.8, \mathrm{H}-5^{\prime} \mathrm{b}\right) ; 4.46$ $\left(d d, 1 \mathrm{H}, \mathrm{J}_{\text {gem }}=12.6, \mathrm{~J}_{5^{\prime} \mathrm{a}, 4^{\prime}}=3.0, \mathrm{H}-5^{\prime} \mathrm{a}\right) ; 4.50\left(\mathrm{ddd}, 1 \mathrm{H}, \mathrm{J}_{4^{\prime}, 3^{\prime}}=4.4, \mathrm{~J}_{4^{\prime}, 5^{\prime}}=3.0,2.8, \mathrm{H}-4^{\prime}\right) ; 5.43$ $\left(\mathrm{dd}, 1 \mathrm{H}, \mathrm{J}_{3^{\prime} 2^{\prime}}=5.6, \mathrm{~J}_{3^{\prime} 4^{\prime}}=4.4, \mathrm{H}-3^{\prime}\right) ; 5.56\left(\mathrm{t}, 1 \mathrm{H}, \mathrm{J}_{2^{\prime}, 1^{\prime}}=\mathrm{J}_{2^{\prime}, 3^{\prime}}=5.6, \mathrm{H}-2^{\prime}\right) ; 6.08\left(\mathrm{~d}, 1 \mathrm{H}, \mathrm{J}_{1^{\prime}, 2^{\prime}}=\right.$ 5.6, H-1'); $7.39\left(\mathrm{~d}, 1 \mathrm{H}, \mathrm{J}_{7,6}=5.7, \mathrm{H}-7\right) ; 8.17(\mathrm{~s}, 1 \mathrm{H}, \mathrm{H}-2) ; 8.35\left(\mathrm{~d}, 1 \mathrm{H}, \mathrm{J}_{6,7}=5.7, \mathrm{H}-6\right)$. ${ }^{13} \mathrm{C} \mathrm{NMR}\left(100.6 \mathrm{MHz} \mathrm{CDCl}_{3}\right): 19.85\left(\mathrm{CH}_{3}\right) ; 20.31,20.50$ and $20.75\left(3 \times \mathrm{CH}_{3} \mathrm{CO}\right) ; 62.73$ $\left(\mathrm{CH}_{2}-5^{\prime}\right) ; 70.10\left(\mathrm{CH}-3^{\prime}\right) ; 73.29\left(\mathrm{CH}-2^{\prime}\right) ; 80.40\left(\mathrm{CH}-4^{\prime}\right) ; 87.05\left(\mathrm{CH}-1^{\prime}\right) ; 104.05$ (CH-7); 136.92 (C-7a); 139.82 (C-3a); 140.39 (CH-2); 142.20 (CH-6); 152.68 (C-4); 169.20, 169.49 and $170.09(3 \times \mathrm{CO})$. IR $\left(\mathrm{CCl}_{4}\right): 1758,1602,1590,1371,1219,1100,1063,1048$. HR MS (ESI): calculated for $\mathrm{C}_{18} \mathrm{H}_{22} \mathrm{~N}_{3} \mathrm{O}_{7}[\mathrm{M}+\mathrm{H}] 392.1458$, found 392.1453 .

\section{4-Ethyl-1-(2,3,5-tri-0-acetyl- $\beta$-D-ribofuranosyl)-1H-imidazo[4,5-c]pyridine (6b)}

An argon purged flask containing a mixture of acetylated 6-chloro-3-deazapurine riboside 3 (288 $\mathrm{mg}, 0.70 \mathrm{mmol}$ ), triethylaluminum ( $1 \mathrm{M}$ solution in hexane; $1.4 \mathrm{ml}, 1.4 \mathrm{mmol}$ ) and $\mathrm{Pd}\left(\mathrm{PPh}_{3}\right)_{4}(40 \mathrm{mg}, 0.035 \mathrm{mmol})$ in THF $(4 \mathrm{ml})$ was stirred at $100{ }^{\circ} \mathrm{C}$ for $3 \mathrm{~h}$. The mixture was diluted with $\mathrm{CHCl}_{3}(20 \mathrm{ml})$ and treated with saturated aqueous $\mathrm{NH}_{4} \mathrm{Cl}(20 \mathrm{ml})$. The slurry was filtered through cellite and after phase separation, aqueous phase was re-extracted with $\mathrm{CHCl}_{3}(2 \times 10 \mathrm{ml})$. Collected organic extracts were dried over $\mathrm{MgSO}_{4}$, volatiles were removed in vacuo and the residue was chromatographed on silica gel (AcOEt) affording product $\mathbf{6 b}$ as colorless oil (169 mg, 60\%). ${ }^{1} \mathrm{H} \mathrm{NMR}\left(400 \mathrm{MHz} \mathrm{CDCl}_{3}\right): 1.44\left(\mathrm{t}, 3 \mathrm{H}, \mathrm{J}_{\text {vic }}=7.6\right.$, $\left.\mathrm{CH}_{3} \mathrm{CH}_{2}\right) ; 2.10,2.17,2.18\left(3 \times \mathrm{s}, 3 \times 3 \mathrm{H}, 3 \times \mathrm{CH}_{3} \mathrm{CO}\right) ; 3.28\left(\mathrm{q}, 2 \mathrm{H}, \mathrm{J}_{\text {vic }}=7.6, \mathrm{CH}_{2} \mathrm{CH}_{3}\right)$; $4.41\left(\mathrm{dd}, 1 \mathrm{H}, \mathrm{J}_{\text {gem }}=12.5, \mathrm{~J}_{5^{\prime} \mathrm{b}, 4^{\prime}}=2.8, \mathrm{H}-5^{\prime} \mathrm{b}\right) ; 4.46\left(\mathrm{dd}, 1 \mathrm{H}, \mathrm{J}_{\mathrm{gem}}=12.5, \mathrm{~J}_{5^{\prime} \mathrm{a}, 4^{\prime}}=3.0, \mathrm{H}-5^{\prime} \mathrm{a}\right)$; $4.50\left(\mathrm{ddd}, 1 \mathrm{H}, \mathrm{J}_{4^{\prime}, 3^{\prime}}=4.3, \mathrm{~J}_{4^{\prime}, 5^{\prime}}=3.0,2.8, \mathrm{H}-4^{\prime}\right)$; $5.43\left(\mathrm{dd}, 1 \mathrm{H}, \mathrm{J}_{3^{\prime} 2^{\prime}}=5.6, \mathrm{~J}_{3^{\prime} 4^{\prime}}=4.3, \mathrm{H}-3^{\prime}\right)$; $5.56\left(\mathrm{t}, 1 \mathrm{H}, \mathrm{J}_{2^{\prime}, 1^{\prime}}=\mathrm{J}_{2^{\prime}, 3^{\prime}}=5.6, \mathrm{H}-2^{\prime}\right) ; 6.09\left(\mathrm{~d}, 1 \mathrm{H}, \mathrm{J}_{1^{\prime}, 2^{\prime}}=5.6, \mathrm{H}-1^{\prime}\right) ; 7.39\left(\mathrm{~d}, 1 \mathrm{H}, \mathrm{J}_{7,6}=5.7\right.$, $\mathrm{H}-7) ; 8.16(\mathrm{~s}, 1 \mathrm{H}, \mathrm{H}-2) ; 8.39\left(\mathrm{~d}, 1 \mathrm{H}, \mathrm{J}_{6,7}=5.7, \mathrm{H}-6\right) .{ }^{13} \mathrm{CNMR}\left(100.6 \mathrm{MHz} \mathrm{CDCl}_{3}\right): 13.22$ $\left(\mathrm{CH}_{3} \mathrm{CH}_{2}\right) ; 20.33,20.50$ and $20.76\left(3 \times \mathrm{CH}_{3} \mathrm{CO}\right) ; 26.85\left(\mathrm{CH}_{2} \mathrm{CH}_{3}\right) ; 62.73\left(\mathrm{CH}_{2}-5^{\prime}\right) ; 70.11$ $\left(\mathrm{CH}-3^{\prime}\right) ; 73.30\left(\mathrm{CH}-2^{\prime}\right) ; 80.37\left(\mathrm{CH}-4^{\prime}\right) ; 87.04\left(\mathrm{CH}-1^{\prime}\right) ; 104.01$ (CH-7); 137.09 (C-7a); 139.20 (C-3a); $140.33(\mathrm{CH}-2) ; 142.32(\mathrm{CH}-6) ; 157.55$ (C-4); 169.22, 169.50 and 170.10 (3 × CO). IR $\left(\mathrm{CCl}_{4}\right): 1757,1600,1588,1371,1220,1103,1063,1048$. HR MS (ESI): calculated for $\mathrm{C}_{19} \mathrm{H}_{24} \mathrm{~N}_{3} \mathrm{O}_{7}[\mathrm{M}+\mathrm{H}]$ 406.1614, found 406.1609.

\section{4-Cyclopropyl-1-(2,3,5-tri-0-acetyl- $\beta$-D-ribofuranosyl)-1H-imidazo[4,5-c]pyridine (6c)}

Tetrahydrofuran $(4 \mathrm{ml}$ ) was added to flame-vacuum dried zinc chloride (340 $\mathrm{mg}, 2.5 \mathrm{mmol}$ ) under argon. The mixture was stirred at $-10{ }^{\circ} \mathrm{C}$ and a solution of cyclopropylmagnesium 
bromide ( $1 \mathrm{M}$ solution in THF; $2.5 \mathrm{ml}$ ) was added dropwise. The mixture was stirred for $40 \mathrm{~min}$ and then a solution of a 6-chloro-3-deazapurine riboside 3 (205 $\mathrm{mg}, 0.5 \mathrm{mmol}$ ) and $\mathrm{Pd}\left(\mathrm{PPh}_{3}\right)_{4}(40 \mathrm{mg}, 0.035 \mathrm{mmol})$ in THF $(5 \mathrm{ml})$ was added. The resulting mixture was stirred at $70{ }^{\circ} \mathrm{C}$ for $8 \mathrm{~h}$. Then the reaction mixture was diluted with water $(50 \mathrm{ml})$ and washed with ethyl acetate $(3 \times 50 \mathrm{ml})$. The collected organic layers were washed with brine, dried over $\mathrm{MgSO}_{4}$ and the residue was purified by column chromatography on silica gel (AcOEt-hexane $0-30 \%)$ providing compound $6 \mathrm{c}$ as a white foam (80 mg, 38\%). ${ }^{1} \mathrm{H} \mathrm{NMR} \mathrm{(500} \mathrm{MHz} \mathrm{CDCl}_{3}$ ): 1.13 and $1.31(2 \times \mathrm{m}, 2 \times 2 \mathrm{H}, \mathrm{H}-2,3$-cycloprop); 2.09, 2.17 and $2.18(3 \times \mathrm{s}, 3 \times 3 \mathrm{H}$, $\mathrm{CH}_{3} \mathrm{CO}$ ); 2.87 (tt, $1 \mathrm{H}, \mathrm{J}_{\text {vic }}=8.3,4.7, \mathrm{H}$-1-cycloprop); $4.41\left(\mathrm{dd}, 1 \mathrm{H}\right.$, J $\mathrm{J}_{\text {gem }}=12.5, \mathrm{~J}_{5^{\prime} \mathrm{b}, 4^{\prime}}=2.9$, $\left.\mathrm{H}-5^{\prime} \mathrm{b}\right) ; 4.45$ (dd, $1 \mathrm{H}$, J $\left.\mathrm{Jgm}_{\mathrm{gem}}=12.5, \mathrm{~J}_{5^{\prime} \mathrm{a}, 4^{\prime}}=3.0, \mathrm{H}-5^{\prime} \mathrm{a}\right) ; 4.49\left(\mathrm{ddd}, 1 \mathrm{H}, \mathrm{J}_{4^{\prime}, 3^{\prime}}=4.3, \mathrm{~J}_{4^{\prime}, 5^{\prime}}=3.0\right.$, 2.9, H-4'); $5.43\left(\mathrm{dd}, 1 \mathrm{H}, \mathrm{J}_{3^{\prime}, 2^{\prime}}=5.6, \mathrm{~J}_{3^{\prime}, 4^{\prime}}=4.3, \mathrm{H}-3^{\prime}\right) ; 5.55\left(\mathrm{dd}, 1 \mathrm{H}, \mathrm{J}_{2^{\prime}, 1^{\prime}}=5.9, \mathrm{~J}_{2^{\prime}, 3^{\prime}}=5.6\right.$, $\left.\mathrm{H}-2^{\prime}\right) ; 6.07\left(\mathrm{~d}, 1 \mathrm{H}, \mathrm{J}_{1^{\prime}, 2^{\prime}}=5.9, \mathrm{H}-\mathrm{I}^{\prime}\right) ; 6.07\left(\mathrm{~d}, 1 \mathrm{H}, \mathrm{J}_{7,6}=5.9, \mathrm{H}-7\right) ; 8.16(\mathrm{~s}, 1 \mathrm{H}, \mathrm{H}-2) ; 8.28(\mathrm{~d}$, $\left.1 \mathrm{H}, \mathrm{J}_{6,7}=5.9, \mathrm{H}-6\right) .{ }^{13} \mathrm{C} \mathrm{NMR}\left(125.7 \mathrm{MHz}, \mathrm{CDCl}_{3}\right): 10.01$ and $10.04\left(\mathrm{CH}_{2}\right.$-2,3-cycloprop); 12.67 ( $\mathrm{CH}$-1-cycloprop); 20.30, 20.50 and $20.75\left(\mathrm{CH}_{3} \mathrm{CO}\right) ; 62.77\left(\mathrm{CH}_{2}-5^{\prime}\right) ; 70.13\left(\mathrm{CH}-3^{\prime}\right)$; 73.26 (CH-2'); $80.38\left(\mathrm{CH}-4^{\prime}\right) ; 86.97\left(\mathrm{CH}^{\prime} \mathbf{1}^{\prime}\right) ; 102.90$ (CH-7); 136.63 (C-7a); 139.52 (C-3a); 140.31 (CH-2); 142.42 (CH-6); 157.06 (C-4); 169.21, 169.52 and $170.14(\mathrm{CO}) . \mathrm{IR}\left(\mathrm{CHCl}_{3}\right)$ : $1591,1372,1232,1199,1096,1067$. HR MS (ESI): calculated for $\mathrm{C}_{20} \mathrm{H}_{24} \mathrm{~N}_{3} \mathrm{O}_{7}[\mathrm{M}+\mathrm{H}]$ 418.1609, found 418.1602 .

\section{4-M ethyl-1-( $\beta$-D-ribofuranosyl)-1H-imidazo[4,5-c]pyridine (5j)}

Compound 6a (129 mg, $0.33 \mathrm{mmol})$ was treated with $1 \mathrm{~m} \mathrm{NaOMe/MeOH} \mathrm{(100} \mathrm{\mu l,} 0.1 \mathrm{mmol})$ in $\mathrm{MeOH}(2 \mathrm{ml})$ at $\mathrm{RT}$ for $1 \mathrm{~h}$ and, after removal of volatiles, the crude product was desalted by reverse phase chromatography and crystallized from water affording nucleoside $\mathbf{5} \mathbf{j}$ as colorless crystals (62 mg, 71\%), m.p. $245-249{ }^{\circ} \mathrm{C}$. $[\alpha]_{D}-68.3$ (c 2.2, DMSO). ${ }^{1} \mathrm{H}$ NMR (400 MHz, DMSO $\left.-d_{6}\right): 2.70\left(\mathrm{~s}, 3 \mathrm{H}, \mathrm{CH}_{3}\right) ; 3.62\left(\mathrm{ddd}, 1 \mathrm{H}, \mathrm{J}_{\mathrm{gem}}=11.9, \mathrm{~J}_{5^{\prime} \mathrm{b}, \mathrm{OH}}=5.1, \mathrm{~J}_{5^{\prime} \mathrm{b}, 4^{\prime}}=3.5, \mathrm{H}-5^{\prime} \mathrm{b}\right)$; 3.67 (ddd, $\left.1 \mathrm{H}, \mathrm{J}_{\text {gem }}=11.9, \mathrm{~J}_{5^{\prime} \mathrm{a}, \mathrm{OH}}=5.3, \mathrm{~J}_{5^{\prime} \mathrm{a}, 4^{\prime}}=3.5, \mathrm{H}-5^{\prime} \mathrm{a}\right) ; 3.99\left(\mathrm{td}, 1 \mathrm{H}, \mathrm{J}_{4^{\prime}, 5^{\prime}}=3.5, \mathrm{~J}_{4^{\prime}, 3^{\prime}}=\right.$ 3.0, $\left.\mathrm{H}-4^{\prime}\right) ; 4.12\left(\mathrm{ddd}, 1 \mathrm{H}, \mathrm{J}_{3^{\prime}, 2^{\prime}}=5.1, \mathrm{~J}_{3^{\prime}, \mathrm{OH}}=4.6, \mathrm{~J}_{3^{\prime}, 4^{\prime}}=3.0, \mathrm{H}-3^{\prime}\right) ; 4.36$ (ddd, $1 \mathrm{H}, \mathrm{J}_{2^{\prime}, \mathrm{OH}}=$ $\left.6.4, \mathrm{~J}_{2^{\prime}, 1^{\prime}}=6.4, \mathrm{~J}_{2^{\prime}, 3^{\prime}}=5.1, \mathrm{H}-2^{\prime}\right) ; 5.16\left(\mathrm{dd}, 1 \mathrm{H}, \mathrm{J}_{\mathrm{OH}, 5^{\prime} \mathrm{a}}=5.3, \mathrm{~J}_{\mathrm{OH}, 5^{\prime} \mathrm{b}}=5.0, \mathrm{OH}-5^{\prime}\right) ; 5.26(\mathrm{~d}, 1 \mathrm{H}$, $\left.\mathrm{J}_{\mathrm{OH}, 3^{\prime}}=4.6, \mathrm{OH}-3^{\prime}\right) ; 5.51\left(\mathrm{~d}, 1 \mathrm{H}, \mathrm{J}_{\mathrm{OH}, 2^{\prime}}=6.5, \mathrm{OH}-2^{\prime}\right) ; 5.88\left(\mathrm{~d}, 1 \mathrm{H}, \mathrm{J}_{1^{\prime}, 2^{\prime}}=6.4, \mathrm{H}-1^{\prime}\right) ; 7.64(\mathrm{~d}$, $\left.1 \mathrm{H}^{\prime} \mathrm{J}_{7,6}=5.7, \mathrm{H}-7\right) ; 8.19\left(\mathrm{~d}, 1 \mathrm{H}, \mathrm{J}_{6,7}=5.7, \mathrm{H}-6\right) ; 8.53(\mathrm{~s}, 1 \mathrm{H}, \mathrm{H}-2) .{ }^{13} \mathrm{C} \mathrm{NMR}(100.6 \mathrm{MHz}$, DMSO-d $\left.{ }_{6}\right): 19.53\left(\mathrm{CH}_{3}\right) ; 61.20\left(\mathrm{CH}_{2}-5^{\prime}\right) ; 70.15\left(\mathrm{CH}-3^{\prime}\right) ; 73.86\left(\mathrm{CH}-2^{\prime}\right) ; 85.78\left(\mathrm{CH}-4^{\prime}\right) ; 88.77$ (CH-1'); 105.29 (CH-7); 137.02 (C-7a); 139.35 (C-3a); 140.96 (CH-6); 142.80 (CH-2); 150.65 (C-4). IR (KBr): 3366, 3254, 1603, 1592, 1493, 1466, 1419, 1314, 1305, 1121, 1092, 1081, 1081, 1045. HR MS (ESI): calculated for $\mathrm{C}_{12} \mathrm{H}_{16} \mathrm{~N}_{3} \mathrm{O}_{4}[\mathrm{M}+\mathrm{H}]$ 266.1141, found 266.1133. For $\mathrm{C}_{12} \mathrm{H}_{15} \mathrm{~N}_{3} \mathrm{O}_{4}$ calculated: $54.33 \% \mathrm{C}, 5.70 \% \mathrm{H}, 15.84 \% \mathrm{~N}$; found: $53.98 \% \mathrm{C}, 5.79 \% \mathrm{H}, 15.51 \% \mathrm{~N}$.

\section{4-Ethyl-1-( $\beta$-D-ribofuranosyl)-1H-imidazo[4,5-c]pyridine (5k)}

Compound $\mathbf{6 b}$ (149 mg, $0.37 \mathrm{mmol})$ was treated with $1 \mathrm{~m} \mathrm{NaOMe} / \mathrm{MeOH}(110 \mu \mathrm{l}, 0.11 \mathrm{mmol})$ in $\mathrm{MeOH}(2 \mathrm{ml})$ at $\mathrm{RT}$ for $1 \mathrm{~h}$ and, after removal of volatiles, the crude product was desalted by reverse phase chromatography and crystallized from water affording nucleoside $\mathbf{5 k}$ as colorless crystals (72 mg, 70\%), m.p. $94{ }^{\circ} \mathrm{C}$. $[\alpha]_{D}-41.0$ (c 1.4, DMSO). ${ }^{1} \mathrm{H} \mathrm{NMR}(400 \mathrm{MHz}$, DMSO- $d_{6}$ ): $1.31\left(\mathrm{t}, 3 \mathrm{H}, \mathrm{J}_{\text {vic }}=7.6, \mathrm{CH}_{3} \mathrm{CH}_{2}\right) ; 3.10\left(\mathrm{q}, 2 \mathrm{H}, \mathrm{J}_{\text {vic }}=7.6, \mathrm{CH}_{2} \mathrm{CH}_{3}\right) ; 3.62(\mathrm{ddd}, 1 \mathrm{H}$, $\left.\mathrm{J}_{\text {gem }}=11.9, \mathrm{~J}_{5^{\prime} \mathrm{b}, \mathrm{OH}}=5.1, \mathrm{~J}_{5^{\prime} \mathrm{b}, 4^{\prime}}=3.5, \mathrm{H}-5^{\prime} \mathrm{b}\right) ; 3.67\left(\mathrm{ddd}, 1 \mathrm{H}, \mathrm{J}_{\mathrm{gem}}=11.9, \mathrm{~J}_{5^{\prime} \mathrm{a}, \mathrm{OH}}=5.3, \mathrm{~J}_{5^{\prime} \mathrm{a}, 4^{\prime}}=\right.$ 3.5, H-5'a); 3.99 (td, $\left.1 \mathrm{H}, \mathrm{J}_{4^{\prime}, 5^{\prime}}=3.5, \mathrm{~J}_{4^{\prime}, 3^{\prime}}=3.0, \mathrm{H}-4^{\prime}\right) ; 4.12\left(\mathrm{ddd}, 1 \mathrm{H}, \mathrm{J}_{3^{\prime}, 2^{\prime}}=5.1, \mathrm{~J}_{3^{\prime}, \mathrm{OH}}=4.6\right.$, $\left.\mathrm{J}_{3^{\prime}, 4^{\prime}}=3.0, \mathrm{H}-3^{\prime}\right) ; 4.37\left(\mathrm{ddd}, 1 \mathrm{H}, \mathrm{J}_{2^{\prime}, \mathrm{OH}}=6.4, \mathrm{~J}_{2^{\prime}, 1^{\prime}}=6.3, \mathrm{~J}_{2^{\prime}, 3^{\prime}}=5.1, \mathrm{H}-2^{\prime}\right) ; 5.16(\mathrm{dd}, 1 \mathrm{H}$, 
$\left.\mathrm{J}_{\mathrm{OH}, 5^{\prime} \mathrm{a}}=5.3, \mathrm{~J}_{\mathrm{OH}, 5^{\prime} \mathrm{b}}=5.0, \mathrm{OH}-5^{\prime}\right) ; 5.26\left(\mathrm{~d}, 1 \mathrm{H}, \mathrm{J}_{\mathrm{OH}, 3^{\prime}}=4.6, \mathrm{OH}-3^{\prime}\right) ; 5.51\left(\mathrm{~d}, 1 \mathrm{H}, \mathrm{J}_{\mathrm{OH}, 2^{\prime}}=6.5\right.$, $\left.\mathrm{OH}-2^{\prime}\right) ; 5.88\left(\mathrm{~d}, 1 \mathrm{H}, \mathrm{J}_{1^{\prime}, 2^{\prime}}=6.3, \mathrm{H}-\mathrm{I}^{\prime}\right) ; 7.64\left(\mathrm{~d}, 1 \mathrm{H}, \mathrm{J}_{7,6}=5.7, \mathrm{H}-7\right) ; 8.22\left(\mathrm{~d}, 1 \mathrm{H}, \mathrm{J}_{6,7}=5.7\right.$, $\mathrm{H}-6) ; 8.52(\mathrm{~s}, 1 \mathrm{H}, \mathrm{H}-2) .{ }^{13} \mathrm{C} \mathrm{NMR}\left(100.6 \mathrm{MHz}\right.$, DMSO-d 6 ): $13.13\left(\mathrm{CH}_{3} \mathrm{CH}_{2}\right) ; 26.11\left(\mathrm{CH}_{2} \mathrm{CH}_{3}\right)$; $61.23\left(\mathrm{CH}_{2}-5^{\prime}\right) ; 70.19\left(\mathrm{CH}-3^{\prime}\right) ; 73.84\left(\mathrm{CH}-2^{\prime}\right) ; 85.81\left(\mathrm{CH}-4^{\prime}\right) ; 88.75\left(\mathrm{CH}-1^{\prime}\right) ; 105.32(\mathrm{CH}-7)$; 137.21 (C-7a); 138.73 (C-3a); 141.03 (CH-6); 142.81 (CH-2); 155.40 (C-4). IR (KBr): 3280, $1605,1593,1493,1477,1415,1312,1217,1119,1087,998$. HR MS (ESI): calculated for $\mathrm{C}_{13} \mathrm{H}_{18} \mathrm{~N}_{3} \mathrm{O}_{4}[\mathrm{M}+\mathrm{H}]$ 280.1297, found 280.1291. For $\mathrm{C}_{13} \mathrm{H}_{17} \mathrm{~N}_{3} \mathrm{O}_{4} \cdot \mathrm{H}_{2} \mathrm{O}$ calculated: $52.52 \% \mathrm{C}$, $6.44 \% \mathrm{H}, 14.13 \% \mathrm{~N}$; found: $52.57 \% \mathrm{C}, 6.46 \% \mathrm{H}, 14.09 \% \mathrm{~N}$.

\section{4-Cyclopropyl-1-( $\beta$-D-ribofuranosyl)-1H-imidazo[4,5-c]pyridine (5I)}

Compound $6 \mathbf{c}(72 \mathrm{mg}, 0.17 \mathrm{mmol})$ was treated with $1 \mathrm{~m} \mathrm{NaOMe} / \mathrm{MeOH}(52 \mu \mathrm{l}, 0.52 \mathrm{mmol})$ in $\mathrm{MeOH}(2 \mathrm{ml}$ ) at $\mathrm{RT}$ for $1 \mathrm{~h}$ and, after removal of volatiles, the crude product was desalted by reverse phase chromatography and crystallized from water- $\mathrm{MeOH}$ affording nucleoside $\mathbf{5 I}$ as colorless crystals (35 mg, 70\%), m.p. $107-113^{\circ} \mathrm{C}$. $[\alpha]_{D}-43.0$ (c $\left.0.23, \mathrm{MeOH}\right) .{ }^{1} \mathrm{H}$ NMR $(500 \mathrm{MHz}$, DMSO- $\left.\mathrm{d}_{6}\right): 1.13$ and $1.22\left(2 \times \mathrm{m}, 2 \times 2 \mathrm{H}, \mathrm{H}-2,3\right.$-cycloprop); $2.86\left(\mathrm{tt}, 1 \mathrm{H}, \mathrm{J}_{\text {vic }}=8.1,4.8\right.$, $\mathrm{H}$-1-cycloprop); 3.72 and $3.77\left(2 \times \mathrm{dd}, 2 \mathrm{H}, \mathrm{J}_{\mathrm{gem}}=12.0, \mathrm{~J}_{5^{\prime}, 4^{\prime}}=3.6, \mathrm{H}-5^{\prime}\right) ; 4.08\left(\mathrm{td}, 1 \mathrm{H}, \mathrm{J}_{4^{\prime}, 5^{\prime}}=\right.$ 3.6, $\left.\mathrm{J}_{4^{\prime}, 3^{\prime}}=3.1, \mathrm{H}-4^{\prime}\right) ; 4.21\left(\mathrm{dd}, 1 \mathrm{H}, \mathrm{J}_{3^{\prime}, 2^{\prime}}=5.2, \mathrm{~J}_{3^{\prime}, 4^{\prime}}=3.1, \mathrm{H}-3^{\prime}\right) ; 4.44\left(\mathrm{dd}, 1 \mathrm{H}, \mathrm{J}_{2^{\prime}, 1^{\prime}}=6.4\right.$, $\left.\mathrm{J}_{2^{\prime}, 3^{\prime}}=5.2, \mathrm{H}-2^{\prime}\right) ; 5.96\left(\mathrm{~d}, 1 \mathrm{H}, \mathrm{J}_{1^{\prime}, 2^{\prime}}=6.4, \mathrm{H}-1^{\prime}\right) ; 7.63\left(\mathrm{~d}, 1 \mathrm{H}, \mathrm{J}_{7,6}=5.6, \mathrm{H}-7\right) ; 8.24(\mathrm{~d}, 1 \mathrm{H}$, $\left.\mathrm{J}_{6,7}=5.6, \mathrm{H}-6\right) ; 8.62(\mathrm{~s}, 1 \mathrm{H}, \mathrm{H}-2) .{ }^{13} \mathrm{C} \mathrm{NMR}\left(125.7 \mathrm{MHz}, \mathrm{DMSO}_{-} \mathrm{d}_{6}\right): 9.84$ and 9.86 $\left(\mathrm{CH}_{2}\right.$-2,3-cycloprop); 12.68 (CH-1-cycloprop); $61.44\left(\mathrm{CH}_{2}-5^{\prime}\right) ; 70.37\left(\mathrm{CH}-3^{\prime}\right) ; 74.14\left(\mathrm{CH}-2^{\prime}\right)$; 86.01 (CH-4'); 89.02 ( $\left.\mathrm{CH}-1^{\prime}\right) ; 104.51$ (CH-7); 137.07 (C-7a); 139.23 (C-3a); 141.47 (CH-6); $143.08(\mathrm{CH}-2)$; 155.14 (C-4). IR (KBr): 3480, 3403, 1601, 1593, 1473, 1455, 1388, 1366, 1221, 1102, 1063, 989, 976. HR MS (ESI) calculated for $\mathrm{C}_{14} \mathrm{H}_{18} \mathrm{~N}_{3} \mathrm{O}_{4}[\mathrm{M}+\mathrm{H}] 292.1292$, found 292.1291. For $\mathrm{C}_{14} \mathrm{H}_{17} \mathrm{~N}_{3} \mathrm{O}_{4} \cdot 1.15 \mathrm{H}_{2} \mathrm{O}$ calculated: $53.89 \% \mathrm{C}, 6.23 \% \mathrm{H}, 13.47 \% \mathrm{~N}$; found: $53.83 \% \mathrm{C}, 6.22 \% \mathrm{H}, 13.43 \% \mathrm{~N}$.

This work is a part of the research project Z4 055 0506. It was supported by the "Centre for New Antivirals and Antineoplastics" (1M0508), by the Programme of Targeted Projects of Academy of Sciences of the Czech Republic (1QS400550501) and by Gilead Sciences, Inc. (Foster City, CA, U.S.A.). Cytostatic activity was studied by Dr I. Votruba (IOCB) and anti-HCV activity by E. Mabery and Dr I. Shih and Dr R. Mackman (Gilead). The contribution of these scientists is gratefully acknowledged.

\section{REFERENCES}

1. a) Hocek M., Holý A., Votruba I., Dvořáková H.: J. Med. Chem. 2000, 43, 1817; b) Hocek M., Holý A., Votruba I., Dvořáková H.: Collect. Czech. Chem. Commun. 2001, 66, 483.

2. Hocek M., Nauš P., Pohl R., Votruba I., Furman P. A., Tharnish P. M., Otto M. J.: J. Med. Chem. 2005, 48, 5869.

3. Hocek M., Holý A., Votruba I., Dvořáková H.: Collect. Czech. Chem. Commun. 2000, 65, 1683.

4. Hocek M., Šilhár P., Pohl R.: Collect. Czech. Chem. Commun. 2006, 71, 1484.

5. Ding Y., Girardet J.-L., Hong Z., Lai V. C. H., An H., Koh Y.-h., Shaw S. Z., Zhong W.: Bioorg. Med. Chem. Lett. 2005, 15, 709. 
6. Fernandez F., Garcia-Mera X., Morales M., Rodriguez-Borges J., De Clercq E.: Synthesis 2002, 1084.

7. Hocek M., Šilhár P., Shih I., Mabery E., Mackman R.: Bioorg. Med. Chem. Lett. 2006, 16, 5290.

8. Hocek M., Holý A., Dvořáková H.: Collect. Czech. Chem. Commun. 2002, 67, 325.

9. Hocek M., Hocková D., Štambaský J.: Collect. Czech. Chem. Commun. 2003, 68, 837.

10. Kimoto M., Moriyama K., Yokoyama S., Hirao I.: Bioorg. Med. Chem. Lett. 2007, 17, 5582.

11. Montgomery J. A., Hewson K.: J. Med. Chem. 1968, 11, 48.

12. Kuchař M., Pohl R., Klepetářová B., Votruba I., Hocek M.: Org. Biomol. Chem. 2008, 6, 2377.

13. Chiang P. K., Richards H. H., Cantoni G. L.: Mol. Pharmacol. 1977, 13, 939.

14. a) review on antiviral properties: Vittori S., Dal Ben D., Lambertucci C., Marucci G., Volpini R., Cristalli G.: Curr. Med. Chem. 2006, 13, 3529; b) Mayers D. L., Mikovits J. A., Joshi B., Hewlett I. K., Estrada J. S., Wolfe A. D., Garcia G. E., Doctor B. P., Burke D. S., Gordon R. K., Lane J. R., Chiang P. K.: Proc. Natl. Acad. Sci. U.S.A. 1995, 92, 215.

15. Endresen P. C., Eide T. J., Aarbakke J.: Biochem. Pharmacol. 1993, 46, 1893.

16. Long M. C., Allan P. W., Luo M.-Z., Liu M.-C., Sartorelli A. C., Parker W. B.: J. Antimicrob. Chemother. 2007, 59, 118.

17. Krenitsky T. A., Rideout J. L., Chao E. Y., Koszalka G. W., Gurney F., Crouch R. C., Cohn N. K., Wolberg G., Vinegar R.: J. Med. Chem. 1986, 29, 138.

18. Mizuno Y., Itoh T., Saito K.: J. Org. Chem. 1964, 29, 2611.

19. Rousseau R. J., Townsend L. B., Robins R. K.: Biochemistry 1966, 5, 756.

20. Vorbrüggen H., Ruh-Pohlenz C.: Org. React. 2000, 55, 1.

21. Sakthivel K., Cook P. D.: Tetrahedron Lett. 2005, 46, 3883.

22. a) Dvořáková H., Holý A., Votruba I., Masojídková M.: Collect. Czech. Chem. Commun. 1993, 58, 629; b) Rousseau R. J., Robins R. K.: J. Heterocycl. Chem. 1965, 2, 196.

23. Western E. C., Daft J. R., Johnson E. M., Gannett P. M., Shaughnessy K. H.: J. Org. Chem. 2003, 68, 6767.

24. a) Čapek P., Hocek M.: Synlett 2005, 3005; b) Čapek P., Pohl R., Hocek M.: Org. Biomol. Chem. 2006, 4, 2278; c) Čapek P., Vrábel M., Hasník Z., Pohl R., Hocek M.: Synthesis 2006, 3515.

25. a) Hirota K., Kitade Y., Kanbe Y., Maki Y.: J. Org. Chem. 1992, 57, 5268; b) Česnek M., Hocek M., Holý A.: Collect. Czech. Chem. Commun. 2000, 65, 1357.

26. a) Šilhár P., Pohl R., Votruba I., Hocek M.: Org. Lett. 2004, 6, 3225; b) Šilhár P., Pohl R., Votruba I., Hocek M.: Collect. Czech. Chem. Commun. 2005, 70, 1669.

27. Lohmann V., Korner F., Koch J., Herian U., Theilmann L., Bartenschlager R.: Science 1999, 285, 110 . 